\title{
Analytic calculations of the spectra of ultra-high energy cosmic ray nuclei. I. The case of $\mathrm{CMB}$ radiation.
}

\author{
R. Aloisio ${ }^{1,2}$, V. Berezinsky ${ }^{2}$ and S. Grigorieva ${ }^{3}$ \\ ${ }^{1}$ INAF, Osservatorio Astrofisico di Arcetri, I-50125 Arcetri (FI), Italy \\ ${ }^{2}$ INFN, Laboratori Nazionali del Gran Sasso, I-67010 Assergi (AQ), Italy \\ ${ }^{3}$ Institute for Nuclear Research, 60th October Revolution Prospect 7A, 117312 Moscow, Russia
}

October 23, 2018

\begin{abstract}
We present a systematic study of different methods for the analytic calculation of ultra-high energy nuclei diffuse spectra. Nuclei propagating in the intergalactic space are photo-disintegrated and decrease their Lorentz factor due to the interaction with cosmic microwave background and extragalactic background light. We calculate the evolution trajectories in the backward time, that describe how atomic mass number $A$ and Lorentz factor $\Gamma$ change with redshift $z$. Three methods of spectra calculations are investigated and compared: (i) trajectory method, (ii) kinetic equation combined with trajectory calculations and (iii) coupled kinetic equations. We believe that these three methods exhaust at least the principal possibilities for any analytic solution of the problem. In the most straightforward method (i) only trajectory calculations are used to connect the observed nuclei flux with the production rate of primary (accelerated) nuclei $A_{0}$. In the second method (ii) the flux (space density) of primary nuclei, and secondary nuclei and protons are calculated with the help of kinetic equation and trajectories are used only to determine the generation rates of these nuclei. The third method (iii) consists in solving the complete set of coupled kinetic equations, written starting with primary nuclei $A_{0}$, then for $A_{0}-1$ etc down to the $A$ of interest. The solution of the preceding equation gives the generation rate for the one which follows. An important element of the calculations for all methods is the systematic use of Lorentz factor instead of energy. We consider here the interaction of nuclei only with the cosmic microwave background, this case is particularly suitable for understanding the physical results. In paper (II) of this series the extragalactic background light will be also included. Estimating the uncertainties of all methods discussed above, we conclude that the method of coupled kinetic equations gives the most reliable results.
\end{abstract}

\section{Introduction}

The observation of particles with ultra high energies $\left(E>10^{18} \mathrm{eV}\right)$ has a fundamental importance in high energy astrophysics and, maybe, more generally in physics. These 
particles, hereafter referred to as Ultra High Energy Cosmic Rays (UHECR), attract much attention in the recent years as the most energetic particles ever observed and because they are messengers from cosmic accelerators to extreme energies.

The observational data on UHECR can be divided into three categories: spectra, mass composition and correlation with astrophysical sources.

Several theoretically well established features are predicted for the energy spectra of UHE protons interacting with the Cosmic Microwave Background (CMB) radiation. Most noticeably these are: (i) the Greisen-Zatsepin-Kuzmin (GZK) feature [1], a sharp steepening of the spectrum at $E \simeq 5 \times 10^{19} \mathrm{eV}$ due to photo-pion production [1]; (ii) a rather faint feature, the dip, at energies $1 \times 10^{18}-4 \times 10^{19} \mathrm{eV}$, caused by the $e^{+} e^{-}$ pair production [2], and (iii) an even more faint and sharp peak at energy $6.3 \times 10^{19} \mathrm{eV}$ produced by an interference effect in the proton interaction with CMB [3].

Two of these features, the GZK cutoff and the dip, most probably are already detected. The observation of the GZK feature in the differential spectrum of HiRes [4] is strengthened by the measured value of $E_{1 / 2}$ in the integral spectrum, which precisely coincides with the theoretical prediction. The recent observations of the Telescope Array detector also confirm the presence of a spectrum steepening consistent with the GZK cut-off [5] The Auger data [6, 7, 8, 9] also show a sharp steepening of the spectrum, which however, according to our calculations, does not fit well the theoretical shape of the GZK feature, especially in the data of 2010 [9] and 2011 [10].

The dip is very well confirmed by the data of Hires, AGASA and Yakutsk detectors [1, 12, 13], and by the Auger data of 2007 [14] though with a larger $\chi^{2}$. The agreement becomes even worse taking the Auger data released in 2010 [9] and the latest release of 2011 [10].

One may notice that both features discussed above are signatures of a pure proton composition. Therefore, agreement of the data with the dip and GZK cutoff, most noticeably in HiRes data, can be considered as an indirect evidence for a proton-dominated composition.

The direct observation of UHECR mass composition is a very difficult task (for a review see [15] and [16]). At present, observations are contradictory. While HiRes [17, Telescope Array [18], HiRes-MIA [19] and Yakutsk [20] detectors favor a proton-dominated flux at energies $E>10^{18} \mathrm{eV}$, Fly's Eye [21], Haverah Park [22], and, recently, Auger [23] indicate a mixed composition with a substantial fraction of heavy nuclei, in particular at the highest energies.

Nowadays the most serious conflict exists between Auger [23] and HiRes [24] data. Both detectors measure the mass composition through the fluorescence emission produced in the atmosphere by UHECR interactions. In particular the quantity that characterizes the mass composition is the position of the maximum of the fluorescence emission, $X_{\max }$, in the atmosphere and its Root Mean Square (RMS) [25]. While HiRes data evidence a pure proton composition till the highest energies, the Auger data show a progressively heavier composition with increasing energy that approaches an almost pure Iron composition at energy $\sim 3.5 \times 10^{19} \mathrm{eV}$.

The third important observable in UHECR is the possible correlation with astrophysical sources. The most energetic UHECR particles can show the direction to nearby sources. This expectation depends on the electrical charge of the particles due to the deflection in surrounding magnetic fields, in particular in the galactic magnetic field. In this case for protons with $E>50 \mathrm{EeV}$ in a microGauss field on homogeneous scale of $\mathrm{Kpc}$ order the deflection angle is around few degrees. With an angular resolution of the 
same order it is possible to resolve UHECR sources on the Mpc scale. For larger electric charge at $E>50 \mathrm{EeV}$, the deflection angle increases up to 10 degrees in the case of Helium and 50 degrees for Iron. Therefore, in the case of nuclei it is difficult to observe any correlation with sources. However, there have been proposed methods to disentangle the effect of the galactic magnetic field on the observed flux, pursuing a correlation study with UHE nuclei [26].

The observational evidences concerning correlations are controversial. The Auger collaboration performed a search for correlation of their events with AGN from the 12th edition of the VCV catalog [27], using the data collected between January 12004 and August 312007 the collaboration found that 20 out of 27 events with energy larger than $(5-6) \times 10^{19} \mathrm{eV}$ correlated with at least one of the selected AGN inside an angle of $3.2^{\circ}$. The updated analysis of such correlation has been performed by the Auger collaboration using the UHECR events collected up to March 312009 with the same selection criteria as before. This updated analysis has not strengthened [28] the signal of correlation with sources as should be expected from an increased statistics.

The Auger results on correlations, coupled with chemical composition, produce an experimental picture with both heavy nuclei and correlation with nearby astrophysical sources at the highest energies, while such correlation is possible only in the case of a large fraction of protons [29].

On the other hand, the HiRes collaboration performed a similar analysis for correlations between stereo UHECR events and AGN from the VCV catalog with no significant correlation found 30].

From a theoretical point of view, there are in literature three models with different predictions for the mass composition. The dip model predicts (almost) a pure proton composition starting from energies $E \geq 10^{18} \mathrm{eV}$ [25]; the mixed composition model [31] favours a mixed composition at the lowest energies with still a proton dominated spectrum at the highest energies and finally the disappointing model explains the observed spectrum in terms of a pure proton composition at $E \sim(1-3) \times 10^{18} \mathrm{eV}$ with steadily heavier mass composition at increasing energy [32].

As shown in [7, the dip and mixed composition models in their present form contradict the Auger data on mass composition. However, the mixed composition model has more power in the explanation of the Auger mass composition: due to presence of several free parameters, the predictions can be, in principle, adjusted to explain a wide range of different mass compositions. In contrast the disappointing model gives the best description of the Auger spectrum and mass composition [32]. On the other hand, the correlations observed by Auger can be accommodated only in the framework of a proton-dominated composition at the highest energies [29, i.e. only in the case of mixed or dip models [29].

For recent reviews on UHECR observations and related models see [33, 34, 35].

The discussion above demonstrates the importance of a theoretical study of nuclei as carriers of the UHE signal. This study has important consequences not only on the interpretation of the observations on mass composition, but also on the spectrum and correlations. The theoretical interest on UHE nuclei is also supported by the difficulties in reaching the highest energies by the acceleration of protons in astrophysical models. In the case of nuclei this problem is ameliorated because the maximum acceleration energy increases by a factor $Z$, the charge number of the nucleus, which is up to 26 in the case of Iron.

Historically, the interest to UHE nuclei started from the hope to solve the UHECR puzzle, i.e. the absence of the GZK cutoff in observations, with the help of nuclei as 
signal carrier. In this case the energy of a CMB photon in the rest system of a nucleus with atomic mass number $A$ is $A$ times lower than for a proton of the same energy, thus photo-pion production is suppressed.

The first calculations of the UHE nuclei photo-disintegration by the CMB radiation have been performed in the works by Stecker [36] and by Puget and Stecker [37], where infrared radiation was also included. Berezinsky and Zatsepin [38] in 1971 have calculated the energy losses of nuclei with different A due to photo-disintegration and pairproduction. It has been demonstrated that steepening in the spectra of heavy nuclei such as Fe, $\mathrm{C}$ etc occurs at a Lorentz-factor determined by the equality of adiabatic and pair-production energy losses, i.e. at energies lower than the GZK cutoff for protons.

The first calculations of the diffuse nuclei spectrum have been made by Berezinsky, Grigorieva, Zatsepin [39] and Hillas [40, both works have been presented at the same conference and published in the same volume of the proceedings.

Propagating in intergalactic space, UHE nuclei interact with the CMB and Extragalactic Background Light (EBL), i.e. Infrared, Visible and Ultra-violet radiation, experiencing two main processes: (i) photo-disintegration and (ii) $e^{+} e^{-}$pair production, that reduce the nucleus kinetic energy. The systematic study of the photo-disintegration started from the pioneering works by Stecker [36, 37, 41, 42]. These works are based on a very convenient parameterization of the photo-nuclear cross-sections, widely used in the study of UHE nuclei propagation. Recently, a refined parameterization of this cross-section was presented in [43].

Using the parameterization of the photo-nuclear cross section by Stecker [41], updated in 1999 [42, or using other approximations as in [43], many papers have been published in the last decade, all (except [44, 45, 46, 47]) based on a Monte Carlo (MC) approach to the study of UHE nuclei propagation in astrophysical backgrounds $[31,42,48,49,50,51$, 52, 53, 554, 55, 556, 57, 558, 59].

The MC computation schemes, typically implemented in these studies, are all based on a statistical treatment only of the process of photo-disintegration, in the case of nuclei, or photo-pion production, in the case of protons, that takes into account the fluctuations associated to the interaction process. The other channel of interaction with astrophysical backgrounds, the pair production process, is usually treated, for both nuclei and nucleons, in the approximation of continuous energy losses, that neglects fluctuations in the interaction assuming that particles loose energy continuously (see later). In the case of photo-pion production for protons this approximation produces a disagreement with the $\mathrm{MC}$ results only at the highest energies and at the level of few percent [60].

The attempts to solve analytically the problem of UHE nuclei propagation are all based on a kinetic-equation approach with the hypothesis of continuous energy losses 44, 45, 46, 47. This approach is very well suited to determine the flux produced by any kind of sources distribution. The approach of [44, 45] is based on a numerical solution of the kinetic (transport) equations for primaries and all secondaries. The results of [44, 45] are particularly interesting for us because represent a numerical test of one of the analytic computation schemes presented here. We will come back to this comparison at the end of the present paper and, more accurately, in the accompanying paper II. The same kineticequation approach is used in the computations presented in [46]. The authors in this case propose a perturbative solution of the transport equation that, at the leading order, takes into account only the process of one nucleon emission in the photo-disintegration process. Finally, in [47] the authors present the complete set of coupled kinetic equations describing the propagation of primary and secondary nuclei; however, their approach is 
focused on the computation of the flux of UHE neutrinos produced by the propagation of CR and doesn't address an explicit computation of the UHE nuclei fluxes.

We present here three new methods for the analytic calculation of UHE nuclei spectra: the evolution-trajectory method, kinetic equation method combined with trajectory calculations and the method of coupled kinetic equations (CKE). In this paper (paper I) we include only CMB as the radiation with which UHE nuclei interact. The main emphasis of this paper is given to the theoretical issues of these methods and comparing them with each other. It is more plausible to do it using the CMB alone, because the Planck spectrum and the exact knowledge of the cosmological evolution of this radiation make formulae simple and the results more transparent. The phenomenological predictions obtained with CMB only are always relevant for the highest energy part of the spectra.

In the accompanying paper II we will include also the interaction with EBL radiations, and focus more on the observational applications of our new computation scheme.

The paper is organized as follows: in section 2 we calculate the energy losses of nuclei on the $\mathrm{CMB}$ radiation, and compute the evolution trajectories. In section 3 the combined method is presented, where space particle density is obtained from kinetic equation, and trajectories are used only for calculations of the generation rates. In section 4 the method of coupled kinetic equations is developed. In section 5 the fluxes obtained with the CKE method are compared with available results in literature. Finally, conclusions are presented in section 6. In the appendixes we give a detailed calculation and discussion of the following technical problems: generation rates calculated from number of particles conservation, in appendix $\mathrm{A}$, the ratio of energy intervals at different redshifts, in appendix B. the analytic solutions of kinetic equations, in appendix $\mathrm{C}$, and the comparison of the secondary nuclei and proton fluxes, in appendix D.

\section{Energy losses and evolution trajectories}

Propagating through background radiations, mainly CMB and EBL, nuclei decrease their Lorentz factor $\Gamma$, due to $e^{+} e^{-}$pair production, and atomic number $A$, due to photodisintegration. Lorentz factor decreases also due to the expansion of the universe (adiabatic energy losses). In this section we study the energy losses and the evolution of nuclei due to their propagation, considering the evolution trajectories in time (or redshift $z$ ) along which $A$ and $\Gamma$ are changing. We will consider these trajectories in the backward time, starting from the point of observation $\left(\Gamma, A, z_{0}=0\right)$ and increasing $z$, so that $\Gamma$ and $A$ increase too.

An important ingredient of our method consists in the use of the Lorentz factor of the particles instead of their energy. This approach gives many simplifications in the theoretical study, in particular the approximate equality of Lorentz factors of all three particles participating in photo-disintegration process $A \rightarrow(A-1)+N$, where $N$ is a nucleon.

\subsection{Energy losses on CMB}

In this paper we consider the interaction of nuclei only with CMB. Adiabatic energy losses and pair production change only the Lorentz factor and photo-disintegration changes only the atomic mass number $A$. Then for $E=\Gamma A m_{N}$ we have

$$
\frac{1}{E} \frac{d E}{d t}=\frac{1}{\Gamma} \frac{d \Gamma}{d t}+\frac{1}{A} \frac{d A}{d t} .
$$


For any process the energy losses in continuous approximation for nuclei or protons interacting with $\mathrm{CMB}$ at $z=0$ can be written as [61]:

$$
\beta_{0}(\Gamma)=-\frac{1}{E} \frac{d E}{d t}=\frac{T}{2 \pi^{2} \Gamma^{2}} \int_{\epsilon_{0}}^{\infty} d \epsilon \sigma(\epsilon) f(\epsilon) \epsilon\left[-\ln \left(1-\exp \left[-\frac{\epsilon}{2 \Gamma T}\right]\right)\right],
$$

where $\epsilon$ is the photon energy in the nucleus or proton rest frame, $\epsilon_{0}$ is the threshold of the considered reaction, $f(\epsilon)$ is the mean fraction of energy in the laboratory system lost by a nucleus in a single interaction, i.e. inelasticity, $\sigma(\epsilon)$ is the cross-section and $T=2.726^{\circ} \mathrm{K}$ is the CMB temperature; units $\hbar=c=k=1$ are used.

At redshift $z$ the number of CMB photons is $(1+z)^{3}$ larger than at $z=0$ and their energies are $(1+z)$ times higher. Then the energy loss at arbitrary $z$ is given by

$$
\beta(\Gamma, z)=(1+z)^{3} \beta_{0}[(1+z) \Gamma]
$$

In the following we will specify the three processes relevant for our calculations: expansion of the universe (adiabatic energy losses), pair production and photo-disintegration.

(i) Adiabatic energy losses.

Adiabatic energy losses are given by

$$
\beta_{\mathrm{ad}}(z)=-\frac{1}{\Gamma} \frac{d \Gamma}{d t}=H(z)
$$

where $H(z)=H_{0} \sqrt{(1+z)^{3} \Omega_{m}+\Omega_{\Lambda}}$ is the Hubble parameter at redshift $z$ with $H_{0}=$ $72 \mathrm{~km} / \mathrm{sMpc}, \Omega_{m}=0.238$ and $\Omega_{\Lambda}=0.716$ according to WMAP data 62].

(ii) Electron-positron pair production

This process can occur if the energy of the background photon is larger than $1 \mathrm{MeV}$ in the rest system of the UHE particle. The inelasticity and cross-section for a nucleus are simply related to the corresponding quantities for the proton pair-production:

$$
f_{\text {pair }}^{A}(\epsilon)=\frac{1}{A} f_{\text {pair }}^{p}(\epsilon), \quad \sigma_{\text {pair }}^{A}(\epsilon)=Z^{2} \sigma_{\text {pair }}^{p}(\epsilon), \quad \beta_{\text {pair }}^{A}(\Gamma)=\frac{Z^{2}}{A} \beta_{\text {pair }}^{p}(\Gamma),
$$

where $Z$ is the nucleus charge number, $m_{N}$ is the nucleon mass, $\sigma_{\text {pair }}^{i}$ is the pair production cross section for protons $(i=p)$ or nuclei $(i=A)$. Using these equations we can rewrite Eq. (2) as

$$
\beta_{\text {pair }}^{A}(\Gamma)=-\frac{1}{\Gamma} \frac{d \Gamma}{d t}=\frac{Z^{2}}{A} \frac{T}{2 \pi^{2} \Gamma^{2}} \int_{\epsilon_{0}}^{\infty} d \epsilon \sigma_{\text {pair }}^{p}(\epsilon) f_{\text {pair }}^{p}(\epsilon) \epsilon\left[-\ln \left(1-\exp \left[-\frac{\epsilon}{2 \Gamma T}\right]\right)\right] .
$$

The expression above is valid for $z=0$. For arbitrary $z$ one should use Eq. (3). In our calculations we use the function $\beta_{\text {pair }}^{p}(\Gamma)$ for protons as computed in [12]. The quantity $\tau_{\Gamma}=\beta_{\text {pair }}^{-1}$ has an important physical meaning being the characteristic time of the Lorentz factor decreasing.

\section{(ii) Photo-disintegration.}

The photo-disintegration energy losses on CMB at $z=0$ are given by

$$
\beta_{\mathrm{dis}}^{A}(\Gamma)=-\frac{1}{A} \frac{d A}{d t}=\frac{T}{2 \pi^{2} \Gamma^{2}} \frac{1}{A} \int_{\epsilon_{0}(A)}^{\infty} d \epsilon \sigma_{\mathrm{dis}}(\epsilon, A) \nu(\epsilon) \epsilon\left[-\ln \left(1-\exp \left[-\frac{\epsilon}{2 \Gamma T}\right]\right)\right],
$$

where $\nu(\epsilon)$ is the average multiplicity of the ejected nucleons. 
The quantity $\beta_{\text {dis }}^{A}$ determines the time-scale of the total photo-disintegration of a nucleus $\tau_{\text {tot }} \sim 1 / \beta_{\text {dis }}^{A}$, while $\tau_{A}=(d A / d t)^{-1}$ gives the mean time for one nucleon loss, or the time between two collisions under an assumption of one-nucleon photo-disintegration $\nu=1$.

At arbitrary $z$ the pair-production energy losses $\beta_{\text {pair }}(\Gamma, z)$ and photo-disintegration energy losses $\beta_{\text {dis }}^{A}(\Gamma, z)$ are given by Eq. (3).

In the present paper we use the remarkable collection of nuclear cross-sections and their parametrization from the works by Stecker et al [41, 42].

Depending on the photon energy $\epsilon$ in the nucleus rest frame, one can distinguish in the general case two different regimes of photo-disintegration, namely the low-energy regime $\epsilon<30 \mathrm{MeV}$ and the high-energy regime: $30<\epsilon<150 \mathrm{MeV}$. At energies $\epsilon>150 \mathrm{MeV}$ the photo-disintegration process is not important [41, 42] and we include at these energies the photo-pion production.

In the low-energy regime the leading process of photo-disintegration is represented by one and two nucleon emission, $A+\gamma \rightarrow(A-1)+N, A+\gamma \rightarrow(A-2)+2 N$, and the photo-disintegration cross-section is dominated by the Giant Dipole Resonance (GDR) 41, 42. At higher energies a multi-nucleon emission regime takes place (see [41, 42]). In this regime the photo-disintegration cross-section can be approximated as constant [41, 42]. The main contribution to photo-disintegration is given by the GDR cross-section with the number of emitted nucleons $\nu=1$ and $\nu=2$.

In the case of CMB radiation considered in the present paper we may limit ourselves by one-nucleon emission only. The typical threshold for two-nucleon emission is $\epsilon_{\mathrm{th}} \sim 20 \mathrm{MeV}$ to be compared with one-nucleon emission threshold $\sim 10 \mathrm{MeV}$. For the Lorentz-factor values of interest $\Gamma \leq 10^{10}$ the photo-disintegration process takes place at the highenergy tail of the Planckian CMB spectrum and two-nucleon production is suppressed by the number of active photons. In fact two-nucleon emission processes are strongly suppressed (by one order of magnitude) at larger Lorentz-factors too by the smallness of the corresponding cross-section (see Fig. 2 from [42]). At extremely large Lorentz-factors the evolution of nuclei enters an explosive regime, described below, for which the difference between one-nucleon and many-nucleons regimes looses any significance. Further on we assume in all formulae below the nucleon multiplicity $\nu=1$, though we keep this quantity in the formulae.

We have calculated the energy losses for all nuclei with existing data, presenting some of them in Figs. 1 and 2 as function of the Lorentz factor.

As discussed below, an important quantity that characterizes nuclei propagation and energy spectra is the critical Lorentz factor $\Gamma_{c}$, defined from the condition of equality between photo-disintegration lifetime $\tau_{A}=(d A / d t)^{-1}$ and the characteristic time $\tau_{\Gamma}$ for Lorentz factor changing,

$$
\tau_{A}\left(\Gamma_{c}\right)=\tau_{\Gamma}\left(\Gamma_{c}\right),
$$

where $\tau_{\Gamma}$ is given by the sum of adiabatic and pair-production losses as $\tau_{\Gamma}^{-1}=\tau_{\text {ad }}^{-1}+\tau_{\text {pair }}^{-1}$.

Eq. (8) is written for $z=0$. The same condition for an epoch $z$ determines $\Gamma_{c}$ at redshift $z$, for which we will use the notation $\Gamma_{c}(z)$.

The values of $\Gamma_{c}$ calculated for some nuclei from Eq. 8 are listed in Table 1. From this Table and Fig. 2 one may notice that $\Gamma_{c}$ is almost the same for all heavy nuclei with a value around $(2-2.5) \times 10^{9}$. The values of $\Gamma_{c}$ calculated from the condition $\beta_{A}\left(\Gamma_{c}\right)=\beta_{\Gamma}\left(\Gamma_{c}\right)$ (see Figs. 1 and 2) differ but little from the values of Table 1 . 

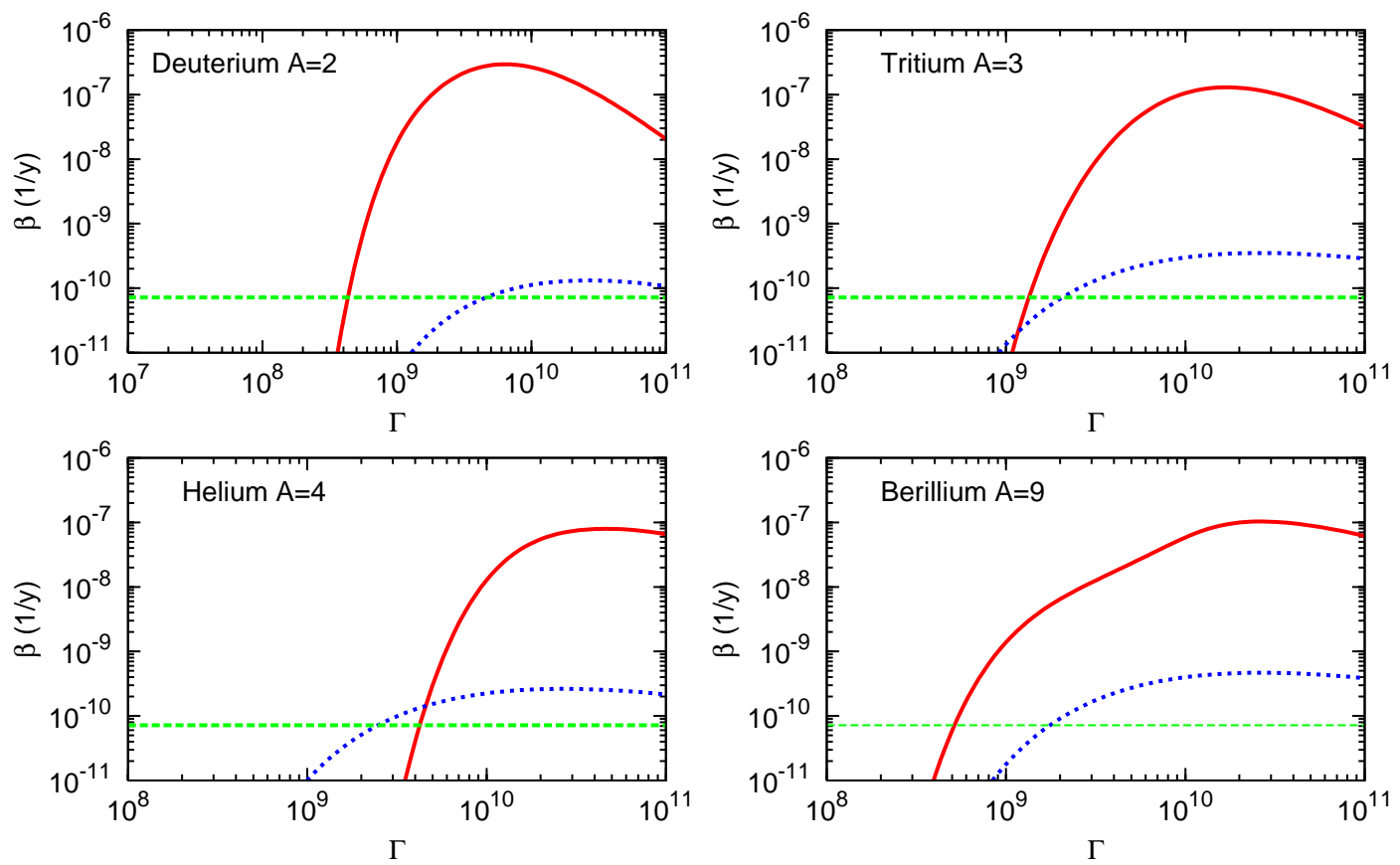

Figure 1: Energy losses for light nuclei due to photo-disintegration and pair production on CMB (red full line and blue dotted line, respectively) and adiabatic energy losses given by $H_{0}$ (green dashed line).
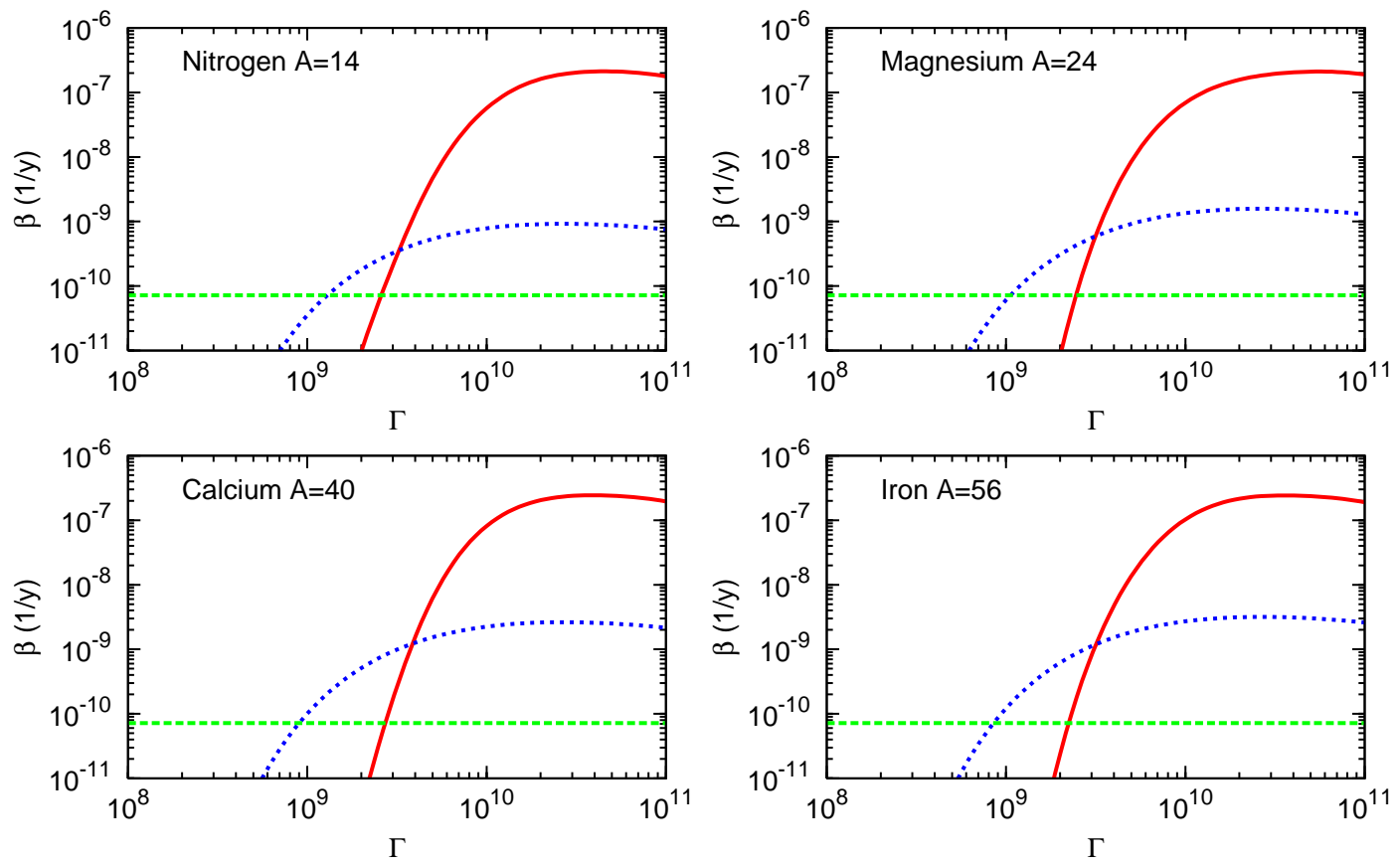

Figure 2: The same as in Fig. 1 for heavy nuclei. 


\begin{tabular}{|c|c|c|c|c|c|c|c|c|}
\hline \hline Nucleus & $D$ & ${ }^{3} \mathrm{He}$ & ${ }^{4} \mathrm{He}$ & ${ }^{9} \mathrm{Be}$ & ${ }^{14} \mathrm{~N}$ & ${ }^{24} \mathrm{Mg}$ & ${ }^{40} \mathrm{Ca}$ & ${ }^{56} \mathrm{Fe}$ \\
\hline$\Gamma_{c}$ & $4 \times 10^{8}$ & $1.2 \times 10^{9}$ & $4 \times 10^{9}$ & $3.8 \times 10^{8}$ & $2.2 \times 10^{9}$ & $2.1 \times 10^{9}$ & $2.3 \times 10^{9}$ & $1.9 \times 10^{9}$ \\
\hline \hline
\end{tabular}

Table 1: Values of $\Gamma_{c}$ for some selected nuclei

\subsection{Evolution trajectories}

A primary nucleus $A_{0}$ accelerated to large Lorentz factor $\Gamma_{g}$ at redshift $z_{g}$ is soon photodisintegrated and then evolves as a secondary nucleus with decreasing $A$ and $\Gamma$ as redshift $z$ decreases.

Our study of the evolution goes in the backward time, whose role is played by redshift. We consider as initial state a secondary nucleus $A$ with Lorentz factor $\Gamma$ and evolve it to larger $z$ with increasing of $A(z)$ and $\Gamma(z)$, until $A(z)$ reaches $A_{0}$ at $z_{g}$ (index $g$ here and henceforth implies generation, i.e. acceleration, of nucleus $\left.A_{0}\right)$. Evolution of $\Gamma$ from $z_{0}=0$ to $z_{g}$ gives $\Gamma_{g}$ of nucleus $A_{0}$. This backward-time evolution is governed by two coupled differential equations, which for the variable $z$ reads

$$
\frac{1}{A} \frac{d A}{d z}=\left|\frac{d t}{d z}\right| \beta_{\text {dis }}(\Gamma, A, z) \quad, \quad \frac{1}{\Gamma} \frac{d \Gamma}{d z}=\left|\frac{d t}{d z}\right| \beta_{\text {pair }}(\Gamma, A, z)+\frac{1}{1+z},
$$

being the relation between time and redshift $d t / d z$

$$
\frac{d t}{d z}=-\frac{1}{(1+z) H(z)}, \quad H(z)=H_{0} \sqrt{(1+z)^{3} \Omega_{m}+\Omega_{\Lambda}}
$$

The term $1 /(1+z)$ in Eq. (9) corresponds to adiabatic energy losses $d \Gamma / d t=-\Gamma H(z)$.

The numerical solution of the coupled equations (9) with initial values $A$ and $\Gamma$ at $z_{0}$ gives the evolution trajectories $A(z)=\mathcal{A}\left(A, \Gamma, z_{0}, z\right)$ and $\Gamma(z)=\mathcal{G}\left(A, \Gamma, z_{0}, z\right)$, where the first three arguments describe the initial condition, in most cases with $z_{0}=0$.

In principle the solution $A(z)$ is given for continuous $A$. We shall refer to the corresponding trajectories as $A$-continuous. In this solution we use $\beta_{\text {dis }}^{A}$ smoothly changing between two integer values of $A$.

In the kinetic-equation approach, which is used for calculation of particle density $n_{A}(\Gamma, z)$, we assume that $A$ is constant until it reaches due to A-evolution $A+1$ (or $A+2)$. The trajectories with this evolution of $A$ we call $A$-jump trajectories. They are calculated assuming in Eq. (9) for $\Gamma$-evolution $A=$ const, until $A$ reaches $A+1$ (or $A+2$ ). Accordingly, we use in this case the jump behaviour of $\beta_{\text {dis }}^{A}$.

In fact, both methods give practically identical results.

A calculated trajectory is sketched in Fig. 3 as $\Gamma(z)=\mathcal{G}(\Gamma, A, z)$ with integer $A$ marked by crosses. In the kinetic equation approach we will interpret this trajectory assuming that the nucleus $A$ is produced in an instantaneous decay of the nucleus $A+1$, then it lives as nucleus $A$ and finally instantaneously decays to $A-1$. The intervals between crosses are determined by continuously changing $A(z)$ setting $\Delta A=1$ (or $\Delta A=2$ ). This is the most natural interpretation, because a nucleus with continuously changing $A$ just does not exist in nature, while instantaneous production of the nucleus $A$ from the decay of $A+1$ is a realistic photo-disintegration assumption, as well as an assumption that after decay of $A+1$ in the forward-running time a nucleus $A$ propagates with fixed $A$ until the instantaneous $A \rightarrow(A-1)$ decay (we always imply below the case of $A+2$ too).

In Fig. 4 the calculated evolution trajectories $\mathcal{A}\left(A, \Gamma, z_{0}, z\right)$ and $\mathcal{G}\left(A, \Gamma, z_{0}, z\right)$ are displayed for the initial condition $A=2$ (Deuterium), $z_{0}=0$ and different values of $\Gamma$, 


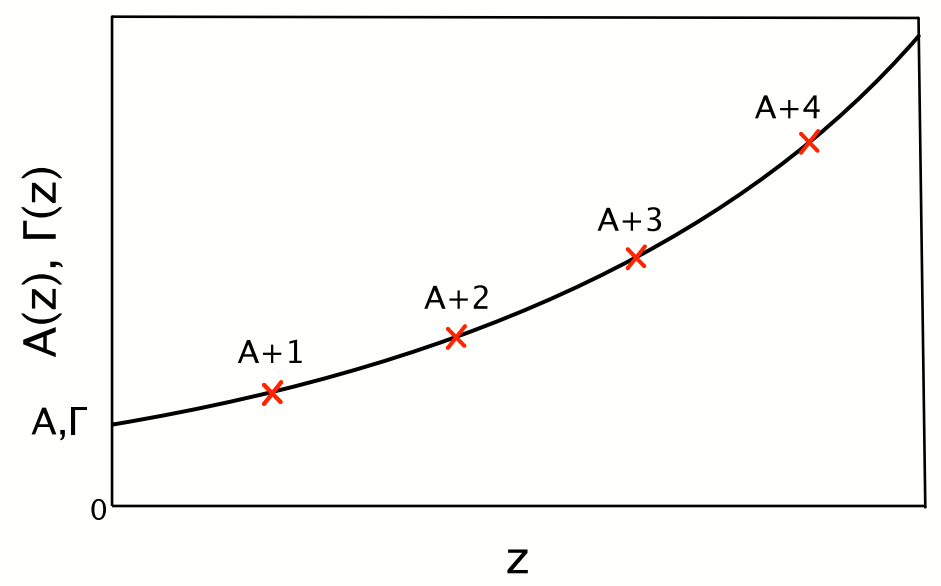

Figure 3: A sketch of $\Gamma(z)$ and $A(z)$ backward-time evolution with $\Gamma(z)$ evolution shown by continuous curve and integer values of $A$ shown by crosses. $\mathrm{A}(\mathrm{z})$ is calculated as continuous quantity only for determination of $z$ where integer values of $\mathrm{A}(\mathrm{z})$ are reached. The transition e.g. from $A$ to $A+1$ nuclei (or $A+2$ ), is assumed to occur instantaneously and marked by crosses. A nucleus between two crosses is considered as one with fixed $A$. Evolution of $\Gamma$ between two crosses is calculated for fixed $A$.
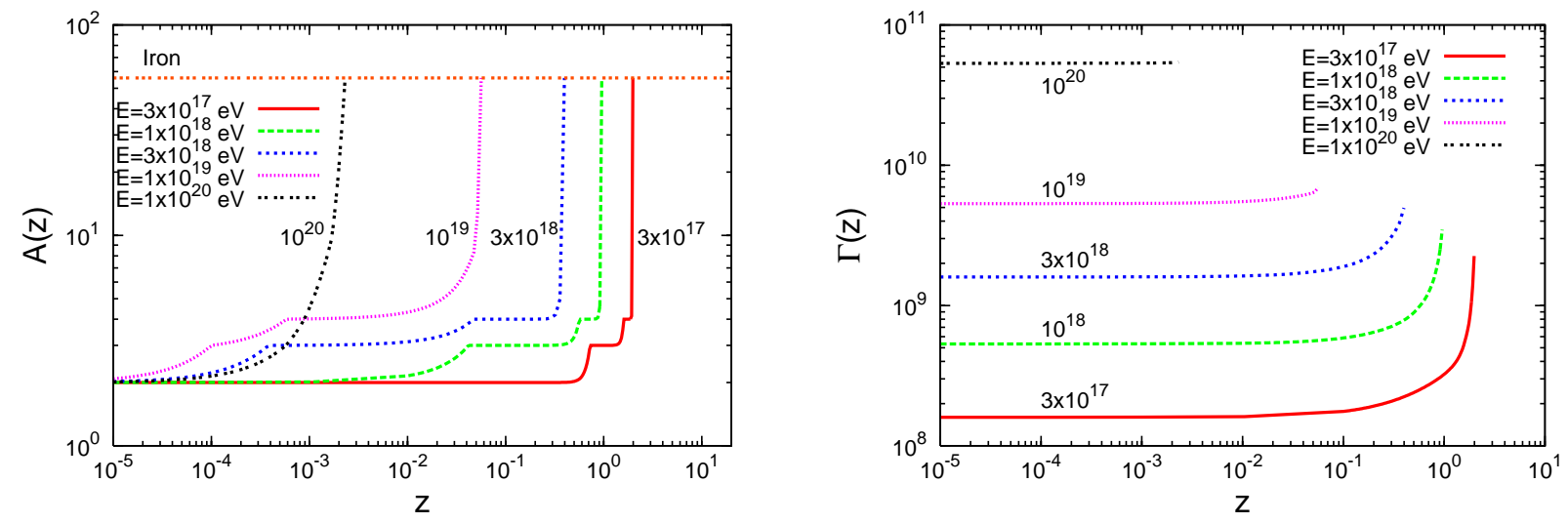

Figure 4: Calculated evolution of the atomic mass number $A(z)$ (left panel) and Lorentz factor $\Gamma(z)$ (right panel) for a deuterium nucleus observed at $z=0$ with various energies as labeled.

corresponding to energy $E$ indicated in the figure. $A(z)$ is evolved to $A_{0}=56$ (Iron) and $z_{g}$ is determined. Then $\Gamma_{g}=\mathcal{G}\left(A, \Gamma, z_{0}, z_{g}\right)$ is calculated as shown by the end points in the right panel of Fig. 4. The trajectories for other values of $A$, e.g. $A=9,14,40$, are very similar. The characteristic feature for all of them is the explosive regime in $A(z)$ evolution at the end of the trajectory. We will discuss it in subsection 2.4.

In the calculations of the evolution trajectories we always include two additional conditions: maximum energy of acceleration $E_{\max }^{\mathrm{acc}}$ (in terms of $\Gamma_{\max }^{\mathrm{acc}}$ ) and nucleus stability restriction. The first restriction is imposed as follows. With $z_{g}$ determined from $\mathcal{A}\left(A, \Gamma, z_{g}\right)=A_{0}$ we calculate $\Gamma_{g}=\mathcal{G}\left(A, \Gamma, z_{g}\right)$. If $\Gamma_{g} \geq \Gamma_{\max }^{\text {acc }}$ this trajectory is forbidden, and the contribution to the flux along this trajectory is set to zero. The second 
compulsory restriction, caused by nucleus stability, is considered in the next subsection.

From the point of view of the final result of our work as calculation of UHE nuclei spectra, the evolution trajectories are needed to different extent. For trajectory method they are the most essential component of calculations. For the combined method the trajectories are needed for calculation of two quantities, $z_{g}$ and $\Gamma_{g}$. The former is calculated from $\mathcal{A}\left(\Gamma, A, z_{g}\right)=A_{0}$ (left panel of Fig. (4)). The latter is calculated as $\Gamma_{g}=\mathcal{G}\left(\Gamma, A, z_{g}\right)$ (the end-points of trajectories in the right panel of Fig. (4). In the coupled kinetic equations the $\mathcal{A}\left(\Gamma, A, z_{g}\right)$ trajectory formally is not included, but it presents implicitly, providing the evolution from $A_{0}$ to $A$.

\subsection{Nucleus stability restriction}

A nucleus $A$ with Lorentz factor $\Gamma<\Gamma_{c}^{A}$ at $z=0$ is stable, i.e. it is not photo-disintegrated during the Hubble time. This is easy to understand from the plots in Fig. 5 ,
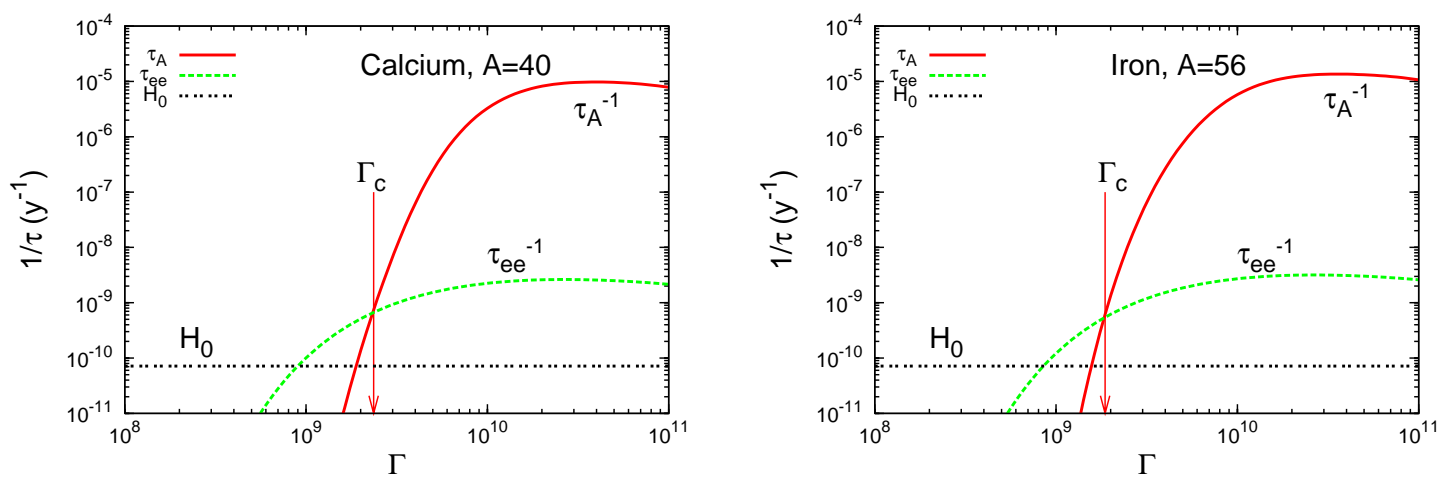

Figure 5: Stability condition for $A=40$ and $A=56$ nuclei. The nucleus $A$ is stable if the decay rate $\tau_{A}^{-1}$ is less than the rate of diminishing the Lorentz factor $\tau_{e e}^{-1}+H_{0}$, where $\tau_{e e}$ is the characteristic time of the pair-production energy losses (see Eq. 8). The critical Lorentz factor given by Eq. (8) coincides approximately with the intersection of $\tau_{A}^{-1}$ and $\tau_{e e}^{-1}$ curves, as shown in the figure.

The critical Lorentz factor is defined by Eq. (8) equating the photo-disintegration lifetime $\tau_{A}=(d A / d t)^{-1}$ and the characteristic time $\tau_{\Gamma}$ for Lorentz factor changing. Consider as an example the forward-in-time evolution of ${ }^{40} \mathrm{Ca}$ nucleus with the Lorentz factor $\Gamma<\Gamma_{c}$. From Fig. 5 it is clear that such nucleus is stable: decreasing of the Lorentz factor goes faster than changing of $A$ and the nucleus slows down, with a further increase of $\tau_{A}(\Gamma)$. Thus, nuclei $A-1$ with $\Gamma<\Gamma_{c}^{A}$ cannot be produced by the decay $A \rightarrow(A-1)+N$ at $z=0$. However, they can be produced at larger $z$.

Consider now the backward-time evolution of the nucleus $(A-1)$ with Lorentz factor $\Gamma<\Gamma_{c}^{A}$. The Lorentz factor of this nucleus increases with $z$ quite fast as $\Gamma(z)=$ $\mathcal{G}\left(A-1, \Gamma, z_{0}, z\right)$, while $A-1$ remains practically unchanged, because $\tau_{A}^{-1} \ll \tau_{\text {pair }}^{-1}$. Simultaneously $\Gamma_{c}^{A}(z)$ decreases with increasing $z$, because the intersection point of $\tau_{A}^{-1}$ and $\tau_{\text {pair }}^{-1}$ is shifted to lower energies by a factor $(1+z)$. As a result at some critical redshift $z_{c}(\Gamma)$, determined by the condition $\tau_{A}\left(\Gamma, z_{c}\right)=\tau_{\Gamma}\left(\Gamma, z_{c}\right)$ the considered nucleus becomes unstable. 
In case of nucleus $A$ produced in $(A+1) \rightarrow A+N$ decay the equation for critical redshift $z_{c}$ becomes

$$
\mathcal{G}\left(A, \Gamma, z_{0}, z_{c}\right)=\Gamma_{c}^{A+1}\left(z_{c}\right),
$$

where $z_{0}=0$. Therefore, to produce the secondary nucleus A with Lorentz factor $\Gamma<$ $\Gamma_{c}^{A+1}$ at $z_{0}=0$, the parent nucleus $A+1$ must decay at $z \geq z_{c}$ determined by the Eq. (11). Thus, $z_{c}(\Gamma)$ can be considered as the minimum red-shift $z_{\min }$ for the production of nucleus A with Lorentz factor $\Gamma<\Gamma_{c}^{A+1}$.

A trajectory for A-nucleus can be blocked not only by $A+1$ nucleus, but also by $A^{\prime}>A+1$, if $A^{\prime}$-nucleus has an anomalously high $\Gamma_{c}$. But in this case the nucleus $A^{\prime}-1$ increases its Lorentz factor with fixed $A^{\prime}-1$ until it reaches $\Gamma_{c}$. Numerical calculations show that stability condition for the considered case puts no restrictions to the calculated trajectories.

\subsection{Explosive regime in $A(z)$ evolution}

The most striking and important feature of $A(z)$ evolution, seen in the left panel of Fig. 4, is its explosive behaviour at the end of the evolution. It is explained by the short lifetime $\tau_{A}$ of nucleus $A$ relative to the transition $A \rightarrow(A+1)$. This lifetime is connected with $\beta_{A}^{0}$, calculated for $z=0$ and plotted in Fig. 2, see also Eq. (3), as

$$
\tau_{A}(\Gamma, z)=(1+z)^{-3} A^{-1} / \beta_{A}^{0}[(1+z) \Gamma] .
$$

This equation demonstrates that $\tau_{A}(\Gamma, z)$ is short and diminishes with $z$. Taking into account that $\sigma_{\text {dis }}$ in Eq. (7) is approximately proportional to $A$ one can understand that $A(t)$ increases exponentially with time. The Lorentz factor at this stage remains constant because the ratio $\tau_{A} / \tau_{\Gamma}$ is less than 100 (see Figs. 11 and 2).

At large Lorentz factors $\Gamma>\Gamma_{c}$ the explosive stage starts at very small $z$ (see the curves $10^{19}$ and $10^{20}$ in the left panel of Fig. (4), and the Lorentz factor during this stage remains constant (see right panel of the figure).
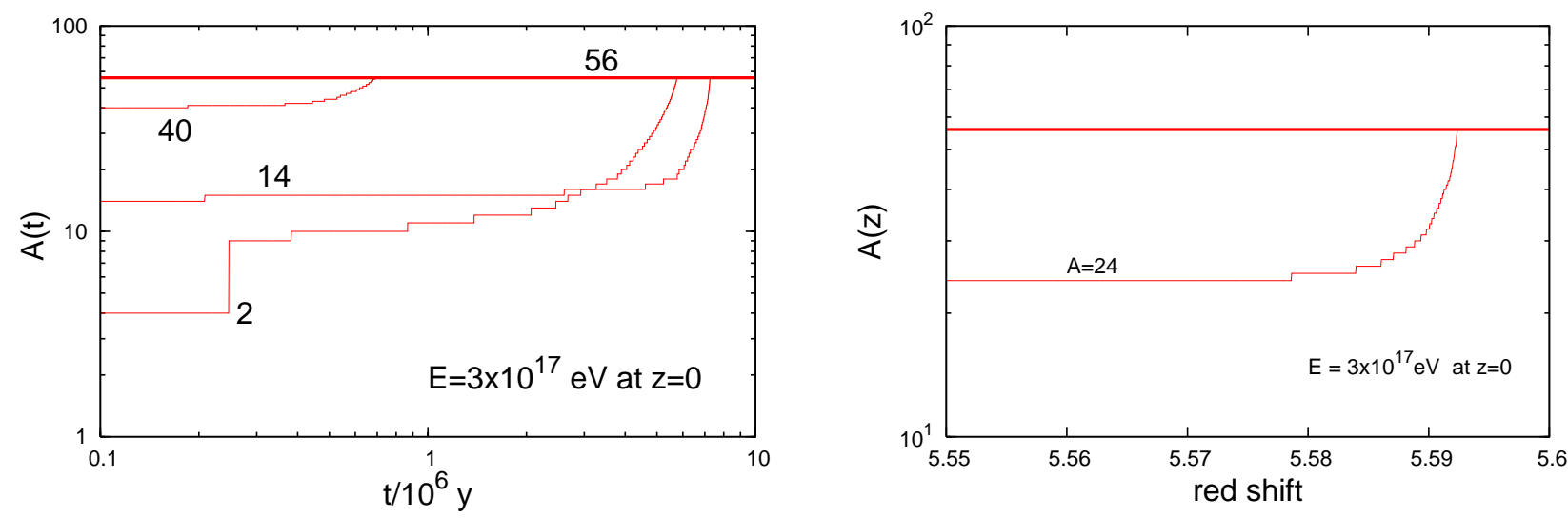

Figure 6: The explosive regimes in the zoomed scale. Left panel: The explosive regime for nuclei with different $A$ and with initial energy $3 \times 10^{17} \mathrm{eV}$ at $z_{0}=0$, shown in a natural time scale. The numbers give $A$. The duration of explosive regime is seen to be short. Right panel: The explosive part of $A(z)$-evolution for $A=24$ and $E=3 \times 10^{17} \mathrm{eV}$ at $z=0$. It starts from $A \rightarrow(A+1)$ transition at $z_{A}=5.58$ and finishes at $z_{g}=5.59$ reaching $A_{0}=56$. 
Let us consider now a nucleus $A$ at $z_{0}=0$ with $\Gamma_{A} \ll \Gamma_{c}^{A+1}$. This case is shown for $\mathrm{A}=2$ in Fig. 4 (left panel) for $\Gamma=1.5 \times 10^{8}$, i.e. $E=3 \times 10^{17} \mathrm{eV}$. From $z_{0}=0$ to $z \sim 0.5$ a nucleus evolves not changing $A$ and changing $\Gamma$ only due to adiabatic energy losses. The critical moment $z_{c}$ occurs when Lorentz factor reaches $\Gamma_{c}$ for ${ }^{3} \mathrm{He}$ according to Eq. (11). Then photo-disintegration starts and almost immediately enters the exponential regime with $\tau_{A}$ given by Eq. (12), which in terms of redshifts is an explosive regime.

The typical cases of explosive trajectories with $\Gamma_{A} \ll \Gamma_{c}^{A+1}$ at $z_{0}=0$ are shown in Fig. 6. In the left panel the explosive regimes for $A=2,14$ and 40 are shown in a natural time scale to demonstrate the short time of evolution. In the right panel the evolution of nucleus $A=24$ is shown for $E=3 \times 10^{17}$ at $z=0$. Until $z=5.58 A$ is not changed, and then during $\Delta z=0.01 A$ jumps to $A_{0}=56$. It illustrates a typical case when the redshift of $A \rightarrow(A+1)$ transition approximately coincides with the redshift of $A_{0}$ appearance, $z_{A} \approx z_{g}$. In this example $z_{A}=5.58$ and $z_{g}=5.59$. Considering then the part of trajectory $A \rightarrow A_{0}$, one obtains $\Gamma_{g}=\mathcal{G}\left(A, \Gamma_{A}, z_{A}, z_{g}\right) \approx \Gamma_{A}$, due to $z_{A} \approx z_{g}$. The relations

$$
z_{A} \approx z_{g}, \quad \Gamma_{A} \approx \Gamma_{g}
$$

are important feature of the explosive trajectories.

For the large Lorentz factors $\Gamma>\Gamma_{c}$, Eq. (13) is naturally fulfilled, too.

\subsection{Fluxes of nuclei and protons in the trajectory method}

In principle trajectory method is similar to MC simulation: both are following trajectory of a nucleus propagating through CMB, calculating the flux of secondary nuclei with Lorentz factor $\Gamma$ from the flux of primary nuclei $A_{0}$ with Lorentz factor $\Gamma_{g}$. As advantage $\mathrm{MC}$ includes fluctuations in the interactions and does not need the introduction of any integration limit.

In the trajectory method the space density $n_{a}\left(\Gamma, z_{0}\right)$, of the components $a=A_{0}, A, p$ is found from the conservation of the number of particles as $n_{a}(\Gamma) d \Gamma=\int d t^{\prime} Q_{a}\left(\Gamma^{\prime}, t^{\prime}\right) d \Gamma^{\prime}$, where $Q_{a}\left(\Gamma^{\prime}, t^{\prime}\right)$ is the generation rate of these particles (see appendix $\underline{\mathrm{A}}$ ). In a way convenient for calculations this formula has the form

$$
n_{a}\left(\Gamma, z_{0}\right)=\int_{z_{\min }}^{z_{\max }} d z^{\prime}\left|\frac{d t}{d z}\right| Q_{a}\left(\Gamma^{\prime}, z^{\prime}\right) \frac{d \Gamma^{\prime}}{d \Gamma}
$$

where $z_{0}=0, Q_{a}\left(\Gamma^{\prime}, z^{\prime}\right)$ is the generation rate of $a$ particles given in appendix $\mathrm{A}$ and $d \Gamma^{\prime} / d \Gamma$ is given in appendix B. The limits of integration are as follows.

For primary nuclei $\left(a=A_{0}\right) z_{\min }=0$ and for $z_{\max }$ we have three options: $z\left(\tau_{A_{0}}\right)$, i.e. redshift corresponding to lifetime $\tau_{A_{0}}(\Gamma), z_{c}(\Gamma)$ as given by Eq. (11), and $z_{g}\left(A_{0}+1\right)$. These three cases give somewhat different results.

For secondary nuclei $(a=A) z_{\min }=0$ for $\Gamma \geq \Gamma_{c}$, and is $z_{c}(\Gamma)$ for $\Gamma \leq \Gamma_{c}$. The maximum limit is given by $z_{A+1}(\Gamma)$.

For secondary protons produced by $(A+1)$ nuclei the limits are the same as for secondary $A$-nuclei, since the production of both is identical (see appendixes $\mathrm{A}$ and $\mathrm{D}$ ).

As numerical calculations show, the trajectory method gives the worst accuracy for flux calculations, especially for secondary nuclei, and this is most probably connected with rather rough estimate of the integration limits, which affect strongly the results. 


\section{Kinetic equation combined with trajectory cal- culations}

In this section we calculate the fluxes (space densities) of primary nuclei, secondary nuclei and protons using the kinetic equation and trajectory calculations. The latter are used in a limited way, only for calculation of generation rate of nuclei and protons, using the number of particles conservation (see appendix $(\mathrm{A})$. In the case of secondary nuclei, the trajectories are used only to determine $\Gamma_{g}$ and $z_{g}$ associated to the primary nucleus $A_{0}$, these calculations may be greatly simplified taking into account that in all practical cases the trajectories are explosive.

We start with the calculations of the secondary-nuclei flux as the most general and technically most interesting part of our calculations.

\subsection{Secondary nuclei}

We consider an expanding universe homogeneously filled by the sources of accelerated primary nuclei $A_{0}$ with a generation rate per unit of comoving volume $Q_{A_{0}}(\Gamma, z)$ given by

$$
Q_{A_{0}}(\Gamma, z)=\frac{\left(\gamma_{g}-2\right)}{m_{N} A_{0}} \mathcal{L}_{0} \Gamma^{-\gamma_{g}}
$$

where $\gamma_{g}>2$ is the generation index, $m_{N}$ is the nucleon mass and $\mathcal{L}_{0}$ is the source emissivity. i.e. the energy generated per unit of comoving volume and per unit time at $z=0$. In Eq. (15) $\Gamma_{\min } \sim 1$ is assumed. In all calculations below we assume also a maximum energy of acceleration $E_{\max }^{\mathrm{acc}}=Z_{0} \times 10^{21} \mathrm{eV}\left(\right.$ or $\left.\Gamma_{\max }^{\operatorname{acc}}=\left(Z_{0} / A_{0}\right) \times 10^{12}\right)$ with the condition $Q_{A_{0}}\left(\Gamma_{g}\right)=0$ at $\Gamma_{g} \geq \Gamma_{\max }^{\text {acc }}$.

The trajectory $A(z)$ is calculated as a continuous quantity only to determine the redshifts of $(A+1) \rightarrow A$ and $A \rightarrow(A-1)$ transitions. Between these values we assume $A=$ const calculating the evolution $\Gamma(z)$ with fixed $A$. Thus, we assume that both of the above-mentioned transitions are instantaneous photo-disintegration, e.g. $\gamma_{\mathrm{CMB}}+(A+1) \rightarrow$ $A+N$. Since a recoil momentum in these processes is negligibly small, one has approximate equality of Lorentz factors of all three particles

$$
\Gamma_{A+1} \approx \Gamma_{A} \approx \Gamma_{N}
$$

The generation rate of secondary nuclei (and secondary nucleons) is derived in appendix $\mathrm{A}$ using the trajectory calculation combined with the conservation of the number of particles

$$
Q_{A}\left(\Gamma_{A}, z\right)=Q_{A_{0}}\left(\Gamma_{g}, z_{g}\right) \frac{1+z}{1+z_{g}} \frac{d \Gamma_{g}}{d \Gamma_{A}}
$$

with $d \Gamma_{g} / d \Gamma_{A}$ given in appendix B,

The kinetic equation for the comoving space density of secondary nuclei $A, n_{A}\left(\Gamma_{A}, t\right)$, reads

$$
\frac{\partial n_{A}\left(\Gamma_{A}, t\right)}{\partial t}-\frac{\partial}{\partial \Gamma_{A}}\left[b_{A}\left(\Gamma_{A}, t\right) n_{A}\left(\Gamma_{A}, t\right)\right]+\frac{n_{A}\left(\Gamma_{A}, t\right)}{\tau_{A}\left(\Gamma_{A}, t\right)}=Q_{A}\left(\Gamma_{A}, t\right),
$$

where $b_{A}=-d \Gamma / d t=\left(\beta_{\text {pair }}+\beta_{\mathrm{ad}}\right) \Gamma_{A}$ is the rate of the Lorentz-factor loss and $Q_{A}\left(\Gamma_{A}, t\right)$ is the generation rate given by Eq. (17), the nucleus $A$ lifetime is 


$$
\tau_{A}^{-1}\left(\Gamma_{A}, z\right)=-d A / d t=\frac{T}{2 \pi^{2} \Gamma^{2}} \int_{\epsilon_{0}(A)}^{\infty} d \epsilon \sigma_{\mathrm{dis}}(\epsilon, A) \nu(\epsilon) \epsilon\left[-\ln \left(1-\exp \left[-\frac{\epsilon}{2 \Gamma T}\right]\right)\right]
$$

where $\nu(\epsilon)$ is the average multiplicity of the emitted nucleons.

The solution of the kinetic equation (18) is found in appendix $\mathrm{C}$ and can be presented as

$$
n_{A}(\Gamma)=\int_{z_{\min }}^{z_{\max }} d z_{A}\left|\frac{d t_{A}}{d z_{A}}\right| Q_{A}\left(\Gamma_{A}, z_{A}\right) \frac{d \Gamma_{A}}{d \Gamma} e^{-\eta\left(\Gamma_{A}, z_{A}\right)}
$$

where $n_{A}(\Gamma)$ is given for $z_{0}=0, z_{A}$ is the redshift of $A$-nuclei production in $A+1 \rightarrow A+N$ decay, $\Gamma_{A}=\mathcal{G}\left(A, \Gamma, z_{0}, z_{A}\right)$ is the Lorentz factor of the nucleus $A$ at the moment of production, calculated using $A=$ const. The quantity $\eta\left(\Gamma_{A}, z\right)$ is given by

$$
\eta\left(\Gamma_{A}, z\right)=\int_{0}^{z} d z^{\prime}\left|\frac{d t^{\prime}}{d z^{\prime}}\right| \frac{1}{\tau_{A}\left(\Gamma_{A}, z^{\prime}\right)}
$$

The physical meaning of the factor $\exp (-\eta)$ becomes clear from Eq. (76) of appendix C. Assuming $\tau_{A}=$ const and introducing as variable a propagation time $t=t_{0}-t^{\prime}$, one obtains this factor as $\exp \left(-t / \tau_{A}\right)$, which gives the survival probability for the nucleus $\mathrm{A}$ during the propagation time $t$.

The factor $\exp (-\eta)$ provides an upper limit of integration in Eq. (20). Without it the trajectory of integration in kinetic equation $\Gamma_{A}(z)$ extends to any large $z$ with the same $A=$ const. Considering this trajectory as the one on which $A$ changes with $z$ too, we can put the marks $z_{A+1}$ and $z_{0}$, which corresponds $A(z)$ reaching $(A+1)$ and $A_{0}$, respectively. From Eq. (21) one can see that at these redshifts the suppression factor $\exp (-\eta)$ is equal to $\exp (-1)$ and $\exp \left[-\left(A_{0}-A\right)\right]$. We shall use $z_{A+1}$ and $z_{A_{0}}$ as trial upper limits in Eq. (20) for comparison with the basic case when the upper limit is set to infinity.

The calculation of the lower limit of integration in Eq. (20) is more complicated. The lower limit $z_{\min }(\Gamma)$ is provided by vanishing of the generation rate $Q_{A}(z)$ at $z \leq z_{\min }$. As has been discussed in section 2.3 at $\mathrm{z}=0$ there is a critical Lorentz factor $\Gamma_{c}^{A+1}$ below which the photo-disintegration of the parent nucleus $A+1$ is absent (see Fig. [5). With increasing $z$ this critical Lorentz factor $\Gamma_{c}^{A+1}(z)$ slightly diminishes because both the pair production and photo-disintegration curves are shifted in Fig. 5 by factor $(1+z)$ to lower energies. If Lorentz factor of a considered $A$-nucleus $\Gamma_{A}<\Gamma_{c}^{A+1}$, the transition $A \rightarrow(A+1)$ at $z=0$ is forbidden and only $\Gamma$ increases with $z\left(\tau_{A}^{-1}\right.$ in Fig. 5 is very small $)$. At $z_{c}$ when the Lorentz factor of $A$-nucleus $\mathcal{G}\left(A, \Gamma, 0, z_{c}\right)$ reaches $\Gamma_{c}^{A+1}\left(z_{c}\right)$ photo-disintegration starts (see Eq. 11). Thus, for $\Gamma<\Gamma_{c}^{A+1} z_{\min }(\Gamma)=z_{c}(\Gamma)$, and for $\Gamma>\Gamma_{c} z_{\min }(\Gamma)=0$.

This effect of $z_{\mathrm{min}}$-appearance can be explained in the evolution-trajectory approach as follows. The production rate of $A$-nuclei, $Q_{A}(z)$, is caused by the decay of $A+1$ nuclei. However, at all $z$ for which $\Gamma_{A+1}(z)<\Gamma_{c}^{A+1}(z)$ a nucleus $A+1$ does not decay and $A+1=$ const, as we observe indeed in the calculation of the trajectories forward in time, and hence $Q_{A}(z)=0$ at $z \leq z_{c}$.

The lower limit $z_{\min }(\Gamma)$ and two trial upper limits are presented in Figs. 7 and 8 , The upper limit $z_{A+1}$ gives the redshift when $A$ formally evolves to $A+1$. The upper limit $z_{A_{0}}$ corresponds to the evolution of $A$ to $A_{0}$. The actual upper limit in this case is provided by the factor $e^{-\eta}$. One may recognize in these figures the regions in the energy-redshift plane which contribute significantly to the flux of the secondary nuclei. In particular, at low energies the interval $z_{\max }-z_{\min }$ tends to zero and the flux vanishes. At the highest 

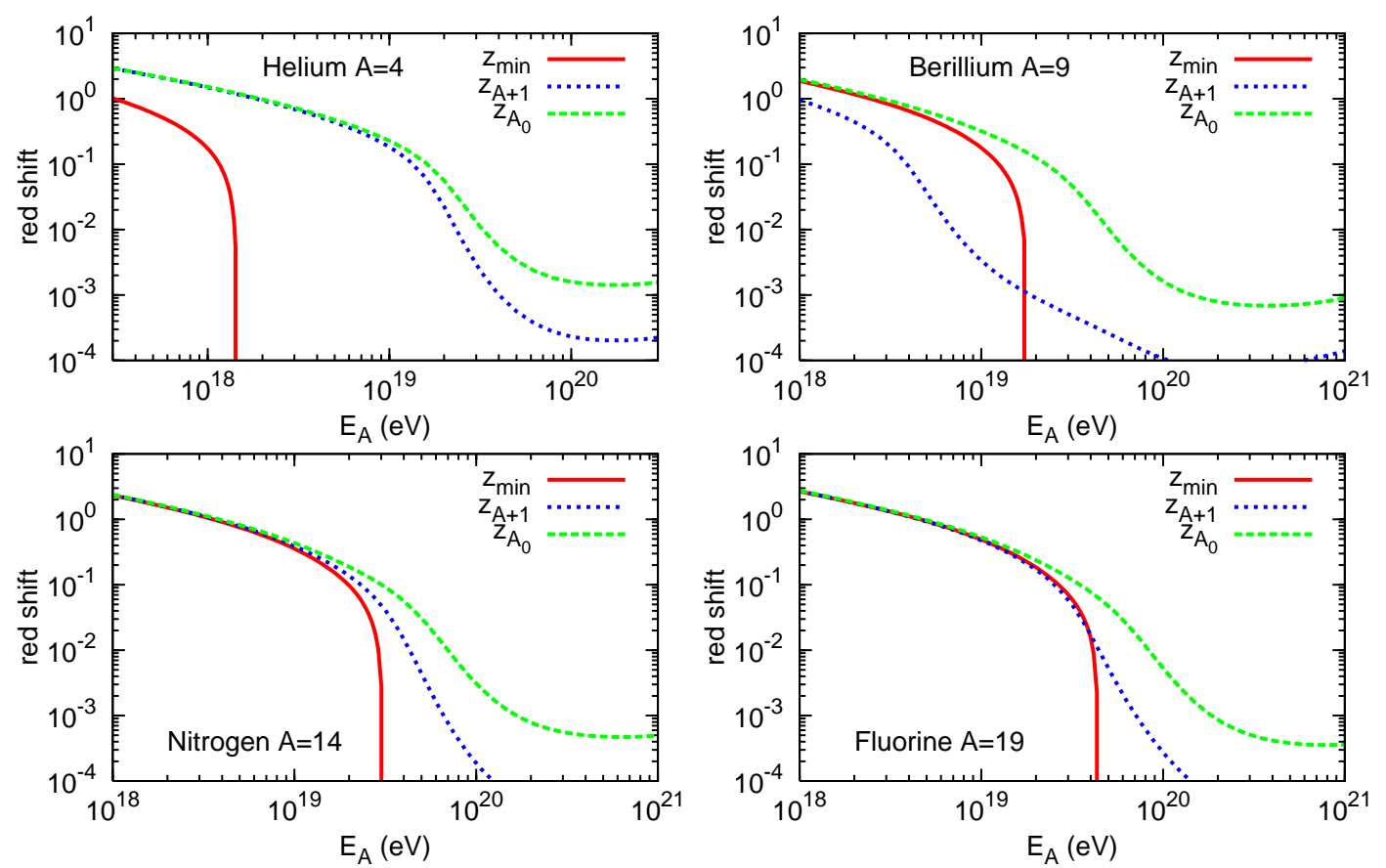

Figure 7: Redshift limits in Eq. (20) for nuclei with small mass number $A$. The values $z_{A+1}$ and $z_{A_{0}}$ correspond to redshifts when the running $A(z)$ reaches $A+1$ and $A_{0}$, respectively.
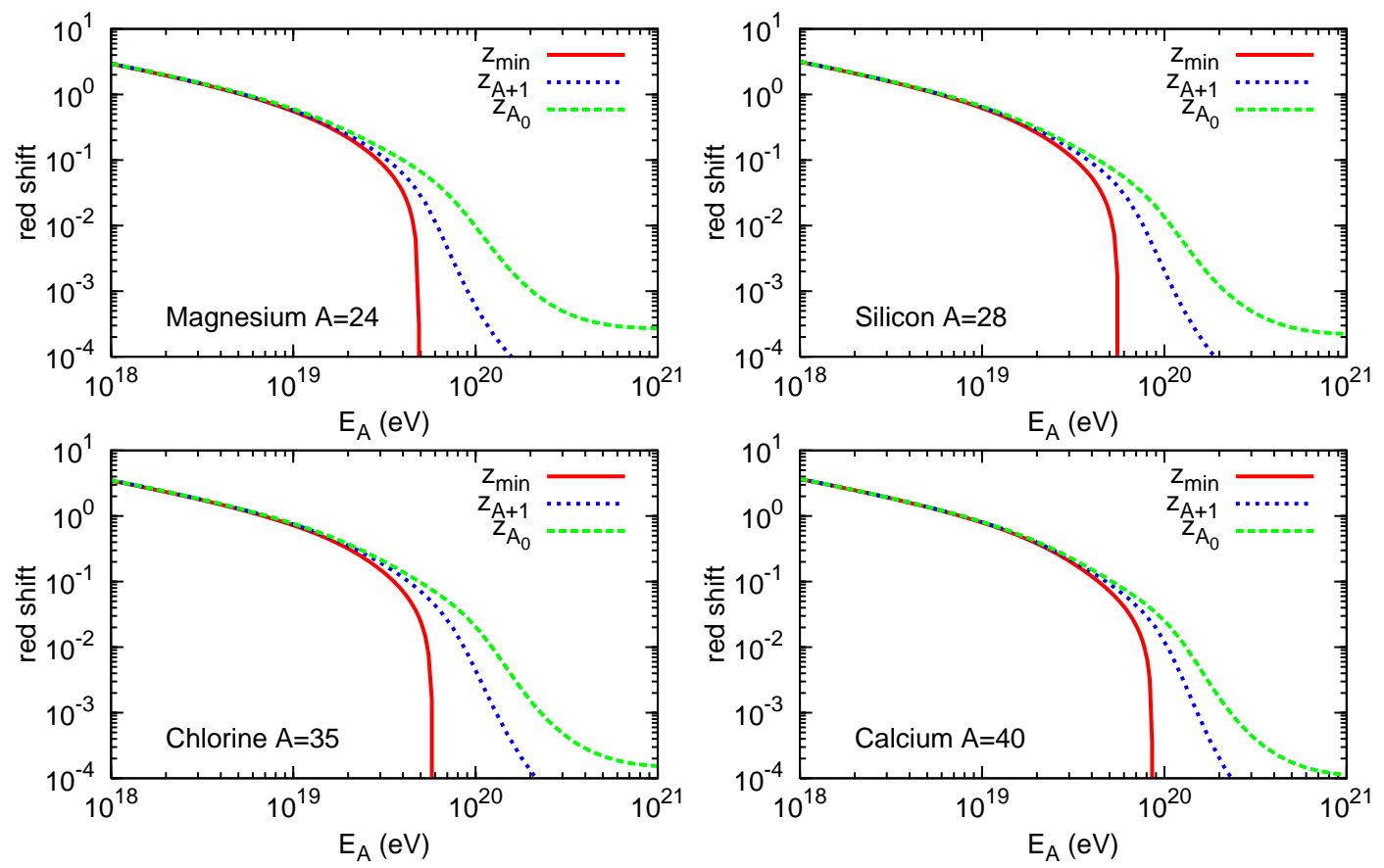

Figure 8: Redshift limits for nuclei with large $A$. Notation is the same as in Fig. 7 , 
energies $E>3 \times 10^{19} \mathrm{eV}$, the interval is widening, but the absolute values of $z_{\text {min }}$ and $z_{\max }$ become vanishingly small, in the range of $10^{-3}$, and thus the fluxes are strongly suppressed.

We will now come over to calculation of fluxes in the form of particle densities $n_{A}(\Gamma, z)$. For this we calculate the generation rates $Q_{A}\left(\Gamma_{A}, z_{A}\right)$ using Eq. (17) and put it into Eq. (20). The left panel in Fig. 9 explains the procedure of integration. The fluxes are shown in Fig. 10 in terms of energy (left panel) and Lorentz factor (right panel).
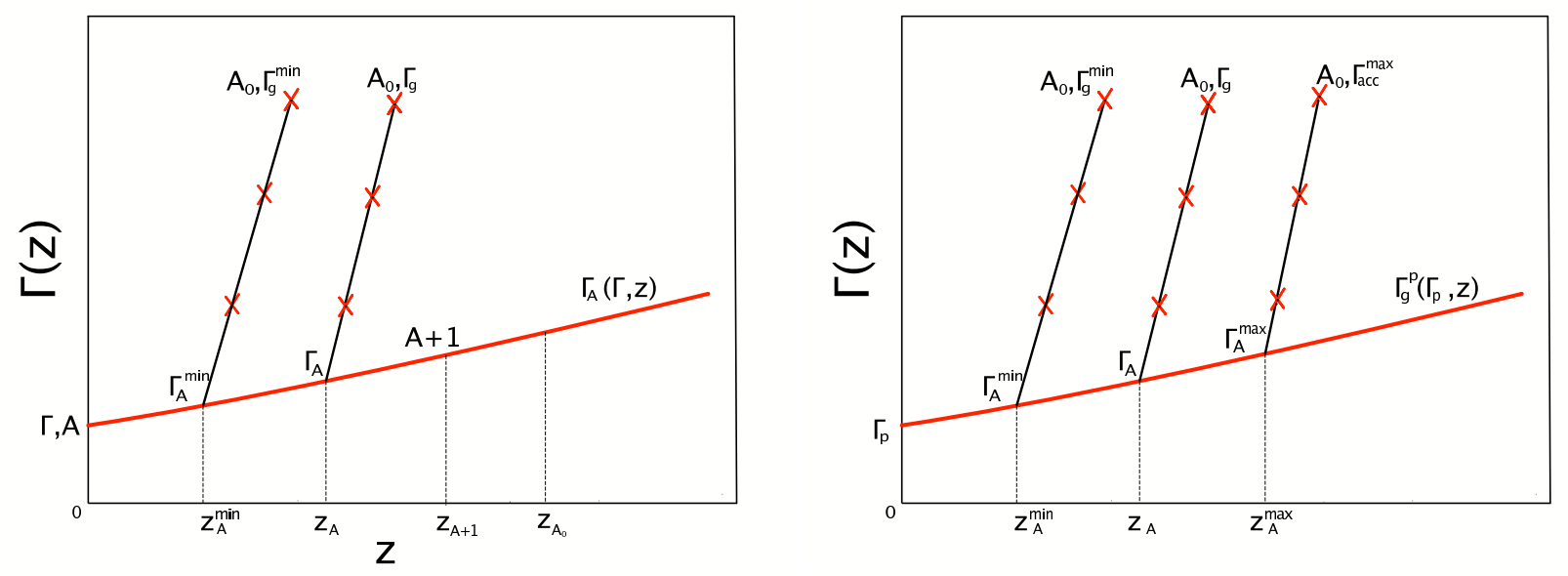

Figure 9: A sketch of $\Gamma(z)$ evolution in kinetic equation approach. Left panel: The thick red line gives the integration trajectory in Eq. (20) described by $\Gamma_{A}\left(\Gamma, z_{0}, z\right)$ with fixed $A=$ const. The auxiliary $A$-evolution trajectories $(A+1) \rightarrow A_{0}$ are needed for calculation of generation rate of $A$-nuclei $Q_{A}\left(\Gamma_{A}, z_{A}\right)$. In practice all these trajectories are explosive, providing $z_{A} \approx z_{g}$ and $\Gamma_{A} \approx \Gamma_{g}$. A point $\left(z_{A}, \Gamma_{A}\right)$ represents the running variables in the integral given by Eq. (20). The lower limit of integration is given by $z_{A}^{\mathrm{min}}$, while $z_{A+1}$ and $z_{A_{0}}$ are the trial upper limits (see text). Right panel related to section 3.2: The thick red line gives the integration trajectory in Eq. (26) for secondary protons. The trajectory describes the Lorentz-factor evolution of a proton $\Gamma_{g}^{p}\left(\Gamma_{p}, z\right)$. Proton is produced at a running point $(z, \Gamma)$ of a proton trajectory in a decay $(A+1) \rightarrow A+N$, and we use in the figure the variables of the brother nucleus $A: z_{A}=z$ and $\Gamma_{A}=\Gamma$. The $A$-evolution sub-trajectories $A \rightarrow A_{0}$ are needed for calculation of generation rate $Q_{p}^{A}\left(\Gamma_{A}, z_{A}\right)$ given by Eq. (27). All these trajectories are explosive, providing $z_{A} \approx z_{g}$ and $\Gamma_{A} \approx \Gamma_{g}$.

One should realize the following feature of calculations: the time scale of $A$ evolution from $(A+1)$ to $A_{0}$ needed for calculation of the rate $Q_{A}\left(\Gamma_{A}, z_{A}\right)$, and the time scale of the kinetic-equation trajectory $\Gamma_{A}(z)$, which contribute the integral in Eq. (20), is the same and very short $\sim \tau_{A}$. In case of $A$-evolution this is the explosive part of the trajectory, in the case of kinetic-equation trajectory it is regulated by $\exp \left(-t / \tau_{A}\right)$. For low energies $E \lesssim 10^{18} \mathrm{eV}$ the lower limit of integration $z_{\min }=z_{c} \approx 2-3$, and $A$ evolution at this $z$ occurs in explosive regime with short evolution time given by Eq. (12). The region of integration in Eq. (20) is controlled by the same $\tau_{A}(\Gamma, z)$. As it is easy to understand the situation is the same at $\Gamma \geq \Gamma_{c}$. In both cases we can use an explosive trajectory for calculation of generation rate, which implies $z_{g} \approx z_{A}$ and $\Gamma_{g} \approx \Gamma_{A}$, and thus Eq. (20) 

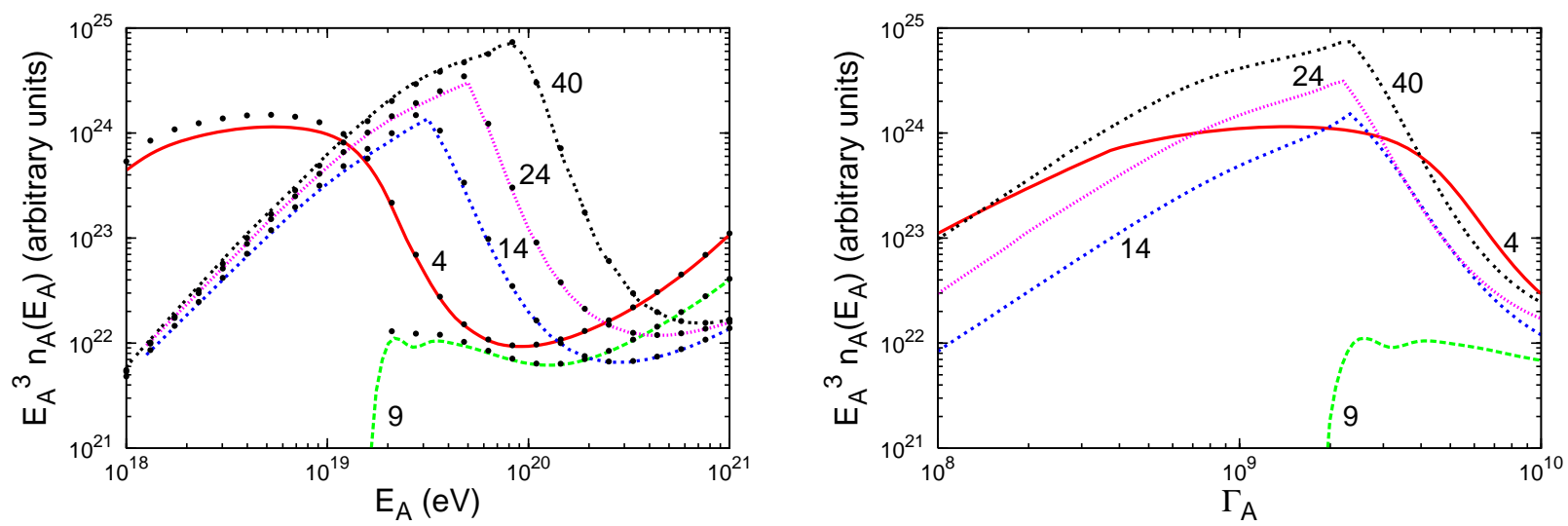

Figure 10: Fluxes of secondary nuclei, as function of energy (left panel) and of the Lorentz factor (right panel). The numbers on the curves show A. The small filled circles present calculations in the explosive trajectory approximation (see the text).

results in

$$
n_{A}(\Gamma)=\frac{\gamma_{g}-2}{m_{N} A_{0}} \mathcal{L}_{0} \int_{z_{\min }(\Gamma)}^{z_{\max }} \frac{d z_{A}}{\left(1+z_{A}\right) H\left(z_{A}\right)} \Gamma_{A}^{-\gamma_{g}} \frac{d \Gamma_{A}}{d \Gamma} e^{-\eta\left(\Gamma_{A}, z_{A}\right)},
$$

where $\Gamma_{A}=\Gamma_{A}\left(\Gamma, z_{0}, z_{A}\right)$ with $A=$ const and $z_{0}=0$.

In Fig. 10 the fluxes calculated in this explosive trajectory approximation are shown by small filled circles. One may observe, as expected, a good agreement with the exact calculations.

The basic parameter which determines the spectra of nuclei $A$ is given by the critical Lorentz factor $\Gamma_{c}^{A+1}$ at which the photo-disintegration process of the parent nucleus $(\mathrm{A}+1$, or Beryllium in the case of Helium) is allowed at $z=0$. One may expect that the peaks seen in the spectra $E_{A}^{3} n_{A}(E)$ set at the critical Lorentz factors and the right panel of Fig. 10 confirms this expectation with a good precision (see Table 1 of section 2.1).

The calculated spectra at low $\Gamma$ exhibit the $A$ hierarchy: the higher $A$ the larger the flux. The exceptional case of low Be flux is explained by large $\tau_{A}$, which determines the upper limit in Eq. (20). In effect, the upper limit $z_{A+1}$ is below $z_{\text {min }}$ (see upper-right panel in Fig. 7), and factor $e^{-\eta}$ in Eq. (20) operates exclusively at large $\eta$ suppressing strongly the Beryllium flux.

The explosive-trajectory approximation allows us to obtain the exact formula for the asymptotic high-energy flux and to compare it with calculations presented in Fig. 10.

In the asymptotic regime one can use in Eq. (22) $\Gamma_{A} \approx \Gamma$ (Lorentz factor is not changing in the pure photo-disintegration process), $z_{\min }=0$ and $z_{g} \ll 1$. Then after simple calculations we obtain

$$
n_{A}(\Gamma)=\left(\gamma_{g}-2\right) \frac{\mathcal{L}_{0}}{A_{0} m_{N}} \Gamma^{-\gamma_{g}} \tau_{A}(\Gamma) .
$$

The asymptotic formula (23) follows also directly from the kinetic equation (18) if one neglects there $\partial n_{A} / \partial t$ and $\partial\left(b_{A} n_{A}\right) / \partial \Gamma_{A}$. Eq. (23) predicts the asymptotic ratio as

$$
n_{A_{1}}: n_{A_{2}}: n_{A_{3} . .}=\tau_{A_{1}}: \tau_{A_{2}}: \tau_{A_{3} . .}
$$


Numerical calculations confirm that asymptotic relations (23) and (24) are fulfilled for the curves in Fig. 10.

For all secondary nuclei at the highest energies the contributing universe is vanishingly small, when the relevant redshifts are of the order of $10^{-3}$ or less, as it follows from curves $z_{A+1}(E)$ in Figs. 7 and 8 . The fluxes in Fig. 10 are non-vanishing only because in the present paper we consider a homogeneous distribution of sources for primary nuclei $A_{0}$ with constant density at any redshift, while in a realistic situation the presence of UHECR sources at these low redshifts $\left(z<10^{-3}\right)$ is unlikely.

The energy spectrum at high energies follows the corresponding energy losses, with a recovery at the highest energy due to an increase of the nucleus lifetime $\tau_{A}$ (i.e. a decrease in the photo-disintegration energy losses, see Figs. 11 and 2).

\subsection{Secondary protons}

In this section we discuss the flux of secondary nucleons produced in the photo-disintegration of the heavier nuclei. We do not need to distinguish neutrons and protons, because at the characteristic lengths involved here neutrons decay fast at all energies of interest. For this reason we will often refer to protons, instead of nucleons.

Production of secondary protons accompanies the production of secondary nuclei, but in contrast to them, protons are not destroyed and can arrive from any redshift, being suppressed only by high energy of generation.

Following the same approach as for secondary nuclei one can write a kinetic equation that describes the propagation of protons:

$$
\frac{\partial n_{p}\left(\Gamma_{p}, t\right)}{\partial t}-\frac{\partial}{\partial \Gamma_{p}}\left[b_{p}\left(\Gamma_{p}, t\right) n_{p}\left(\Gamma_{p}, t\right)\right]=Q_{p}\left(\Gamma_{p}, t\right)
$$

where $n_{p}$ is the secondary-proton density, $b_{p}=-d \Gamma / d t$ describes the loss of proton Lorentz factor (energy loss) due to adiabatic energy losses, pair-production and photo-pion production on the CMB radiation, and $Q_{p}$ is the generation rate for secondary protons, produced by photo-disintegration of secondary or primary nuclei. The secondary nucleons $N$ which accompany production of $A$-nuclei in the process $A+1 \rightarrow A+N$ have the same Lorentz factor and generation rate as A-nuclei. We refer to these nucleons as " $A$ associating protons" with notation $n_{p}^{A}$ for their space density, and use for their generation rate $Q_{p}^{A}(\Gamma, t)=Q_{A}(\Gamma, t)$. The solution of Eq. (25) for $A$-associating protons similarly to 63], reads:

$$
n_{p}^{A}\left(\Gamma_{p}\right)=\int_{z_{\min }}^{z_{\max }} d z^{\prime}\left|\frac{d t^{\prime}}{d z^{\prime}}\right| Q_{p}^{A}\left(\Gamma^{\prime}, z^{\prime}\right)\left(\frac{d \Gamma^{\prime}}{d \Gamma_{p}}\right)_{p},
$$

where $\Gamma^{\prime}\left(z^{\prime}\right)=G_{p}\left(\Gamma_{p}, 0, z^{\prime}\right)$ and $d \Gamma^{\prime} / d \Gamma_{p}$ is taken along the proton trajectory $\left(\Gamma_{g}^{p}\left(\Gamma_{p}, z\right)\right.$ line in the right panel of Fig. 9).

As in Eq. (17) we have from the conservation of particles number:

$$
Q_{p}^{A}\left(\Gamma^{\prime}, z^{\prime}\right)=Q_{A_{0}}\left(\Gamma_{g}, z_{g}\right) \frac{1+z^{\prime}}{1+z_{g}}\left(\frac{d \Gamma_{g}}{d \Gamma^{\prime}}\right)_{A},
$$

where the redshift of $A+1$ decay $z_{A}=z^{\prime}$ and $\Gamma_{A}=\Gamma^{\prime}$, index $A$ at derivative $d \Gamma_{g} / d \Gamma^{\prime}$ indicates the $A$-variable subtrajectory $A \rightarrow A_{0}$ for which derivative is given by Eq. (68), and $Q_{A_{0}}$ is the generation (acceleration) rate of primaries $A_{0}$ at redshift $z_{g}$ with Lorentz factor $\Gamma_{g}$, given by Eq. (15). The generation redshift $z_{g}$ is determined from $\mathcal{A}\left(A, \Gamma^{\prime}, z^{\prime}, z_{g}\right)=A_{0}$ 
and the generation Lorentz factor is calculated as $\Gamma_{g}=\mathcal{G}\left(A, \Gamma^{\prime}, z^{\prime}, z_{g}\right)$. Finally, we obtain for $n_{p}^{A}\left(\Gamma_{p}, z_{0}\right)$ at $z_{0}=0$ :

$$
n_{p}^{A}\left(\Gamma_{p}\right)=\frac{\gamma_{g}-2}{A_{0} m_{N}} \mathcal{L}_{0} \int_{z_{\min }}^{z_{\max }} d z^{\prime}\left|\frac{d t^{\prime}}{d z^{\prime}}\right| \Gamma_{g}^{-\gamma_{g}} \frac{1+z^{\prime}}{1+z_{g}}\left(\frac{d \Gamma_{g}}{d \Gamma^{\prime}}\right)_{A}\left(\frac{d \Gamma^{\prime}}{d \Gamma_{p}}\right)_{p},
$$

the integration in Eq. (28) goes along the proton trajectory $\Gamma_{g}^{p}\left(\Gamma_{p}, z\right)$, i.e $G_{p}\left(\Gamma_{p}, 0, z\right)$, as sketched in the right panel of Fig. 9. A proton can be produced at any running point of this trajectory $\left(z_{A}, \Gamma_{A}\right)$, with $z_{A}=z^{\prime}$ and $\Gamma_{A}=\Gamma^{\prime}$, in a decay $(A+1) \rightarrow A+N$. The generation rate at such point $Q_{p}^{A}\left(z_{A}, \Gamma_{A}\right)$ is calculated using Eq. (27) along the $A$ evolution subtrajectory $A \rightarrow A_{0}$. For the different subtrajectories we have different values of $\Gamma_{g}$ for the primary nucleus $A_{0}$ and different redshifts of generation $z_{g}$.

The minimum redshift in Eq. (28) is calculated as explained in sections 2.3 and 3.1. If the proton Lorentz factor $\Gamma_{p}<\Gamma_{c}^{A+1}(z=0)$, the minimum redshift corresponds to $z_{c}$ at which the proton Lorentz factor $\Gamma_{g}^{p}\left(z_{c}\right)$ reaches the critical Lorentz factor of the $(A+1)$ nucleus $\Gamma_{c}^{A+1}\left(z_{c}\right)$. Note that $z_{c}=z_{\text {min }}$ for protons are higher than for secondary nuclei (compare Fig. [1] with Figs. 7 and 8). It happens because $\beta_{\text {pair }}^{A}(\Gamma)$ is $Z^{2} / A \approx A / 4$ of that for proton (see Eq. 6). In the case $\Gamma_{p}>\Gamma_{c}, z_{\min }=0$. The maximum upper limit $z_{\max }$ corresponds to $A(z)$ subtrajectory on which the Lorentz factor of the $A_{0}$-nucleus reaches $\Gamma_{\text {acc }}^{\max }$ (see Fig. 9). These limits are plotted in Fig. 11, with $z_{\max }$ shown by solid red curve. The other upper limit shown by small filled circles is valid for the instantaneous method (see below). Here it is important to note that both limits coincide with great accuracy.

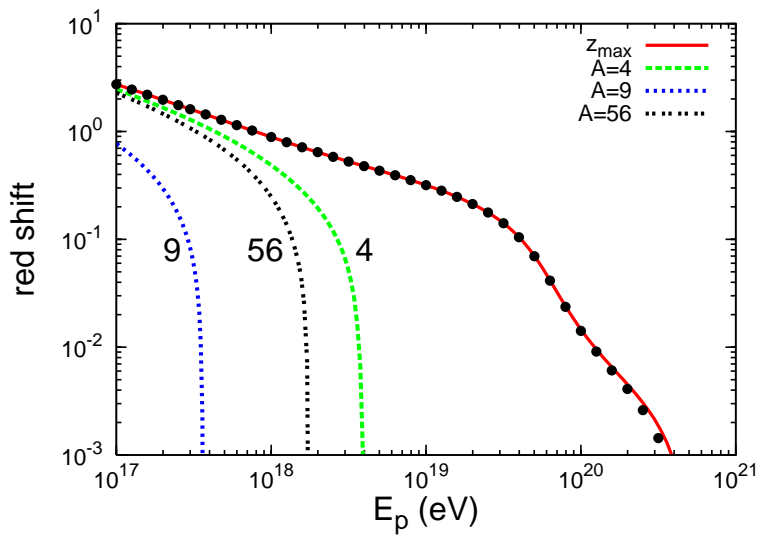

Figure 11: Upper and lower redshift limits in Eq. (28). The lower limits are shown for three proton-associating nuclei with $\mathrm{A}=4,9$ and 56 and labelled by these numbers. The highest limit for Helium and the lowest for Beryllium are explained by the highest and lowest critical Lorentz factors $\Gamma_{c}$ for these nuclei. The lower limits for $A=14,24,35$ and 40 (not shown here) are grouped between $\mathrm{A}=4$ and 56. The upper limit (see text) is given by full red curve. The small filled circles show the upper limit for the instantaneous approach.

The total space density of secondary protons is given by the summation over the primary $A_{0}$ and all the secondaries $A$ in Eq. (28):

$$
n_{p}\left(\Gamma_{p}\right)=\sum_{A \leq A_{0}} n_{p}^{A}\left(\Gamma_{p}\right)
$$


Before discussing the numerical results for the calculated flux, we present an alternative and more simple method of calculation, which, being less precise, can be considered as a test at high energies for the calculations described above.

This approach will be referred to as "primary-nucleus instantaneous decay". It is based on the assumption that at the moment of production $z_{g}$ the primary nucleus is instantaneously photo-disintegrated to $A_{0}$ nucleons. At large Lorentz factors this assumption is well justified because the nucleus lifetime $\tau_{A_{0}}\left(\Gamma_{g}\right)$ is much shorter than all other relevant time scales of the problem. We will demonstrate below why this approach works well (with some exceptions) at lower Lorentz factors $\Gamma<\Gamma_{c}$ and $\Gamma \ll \Gamma_{c}$. To distinguish the previous more precise approach from the instantaneous one, we will refer to the former as intermediate A-decay method.

The proton space density in the instantaneous approach can be written as

$$
n_{p}^{\text {inst }}\left(\Gamma_{p}\right)=A_{0} \int_{z_{g}^{\min }\left(A_{0}\right)}^{z_{g}^{\max }} d z_{g}\left|\frac{d t_{g}}{d z_{g}}\right| Q_{A_{0}}\left(\Gamma_{g}^{p}, z_{g}\right) \frac{d \Gamma_{g}^{p}}{d \Gamma_{p}}
$$

where all notations are as before and $Q_{A_{0}}$ are given by Eq. (15).

Since $Q_{A_{0}} \propto 1 / A_{0}$, the factor $A_{0}$ disappears from Eq. (30) and $n_{p}^{\text {inst }}\left(\Gamma_{p}\right)$ can depend on $A_{0}$ only through the limits of integration.

After a simple rearrangement of Eq. (30) one obtains

$$
n_{p}^{\text {inst }}\left(\Gamma_{p}\right)=\frac{\mathcal{L}_{0}}{H_{0}} \frac{\gamma_{g}-2}{m_{N}} \int_{z_{g}^{\min }}^{z_{g}^{\max }} d z_{g} \frac{\left[\Gamma_{g}^{p}\left(\Gamma_{p}, z_{g}\right)\right]^{-\gamma_{g}}}{\left(1+z_{g}\right) \sqrt{\Omega_{m}\left(1+z_{g}\right)^{3}+\Omega_{\Lambda}}} \frac{d \Gamma_{g}^{p}}{d \Gamma_{p}} .
$$

Eq. (31) is very simple for calculations, because it involves only the proton trajectory in $\Gamma-z$ plane (subtrajectories $A \rightarrow A_{0}$ in the right panel of Fig. 9 shrink to a point). The limits of integration are the same as those shown in Fig. 11, The basic observation involved in the calculation of these limits is that the $A_{0}$ nucleus is now located on the proton trajectory $\Gamma_{g}^{p}\left(\Gamma_{p}, z\right)$ in Fig. 9. Therefore, $z_{g}^{\min }$ is defined by the equation $\Gamma_{g}^{p}\left(\Gamma_{p}, z_{\min }\right)=$ $\Gamma_{c}^{A_{0}}\left(z_{\text {min }}\right)$, which is the same as for nuclei $A$, considered now as $A_{0}$ in Fig. 11. The upper limit is defined by the condition $\Gamma_{g}^{p}\left(\Gamma_{p}, z_{\max }\right)=\Gamma_{\text {acc }}^{\max }$ and it is shown in Fig. 11 by small filled circles, which coincides with upper limit in the intermediate A method. We see, thus, that the upper limit $z_{\max }$ does not depend on $A_{0}$. The lower limit at $\Gamma_{p} \geq \Gamma_{c}^{A_{0}}$ is $z_{\min }=0$. At $\Gamma_{p} \leq \Gamma_{c}$ the lower limit depends on $A_{0}$, but weakly, with the exceptional case of Beryllium.

Thus, at high energy when $z_{\min }=0$ the flux has a universal form independent of the primary nucleus, i.e. of $A_{0}$. It is natural to expect that the high energy regime starts from $\Gamma_{p}>\Gamma_{c}^{A_{0}}$ when $z_{\min }=0$, and the calculations below confirm this expectation.

The high-energy regime is of particular interest as a test of the intermediate A-decay method. In this case the proton flux in instantaneous approximation $n_{p}^{\text {inst }}$ must coincide with the intermediate $A$ method, when the flux is given by $\sum n_{p}^{A}$, i.e. by Eq. (29). It follows also from Figs. 11 and 2, which show that at $\Gamma>\Gamma_{c}$ the photo-disintegration rate strongly dominates over the rate of the Lorentz-factor loss. Figs. 12 and 13 reliably confirm the agreement of both calculations at $\Gamma>\Gamma_{c}$, demonstrating thus that the intermediate $A$ method has passed this test.

We address now the question of the agreement between A-intermediate and instantaneous methods at $\Gamma<\Gamma_{c}$ and $\Gamma \ll \Gamma_{c}$. The explanation follows from the explosive character of $A(z)$ trajectories for $A \rightarrow A_{0}$ evolution. Using the explosive-trajectory result 

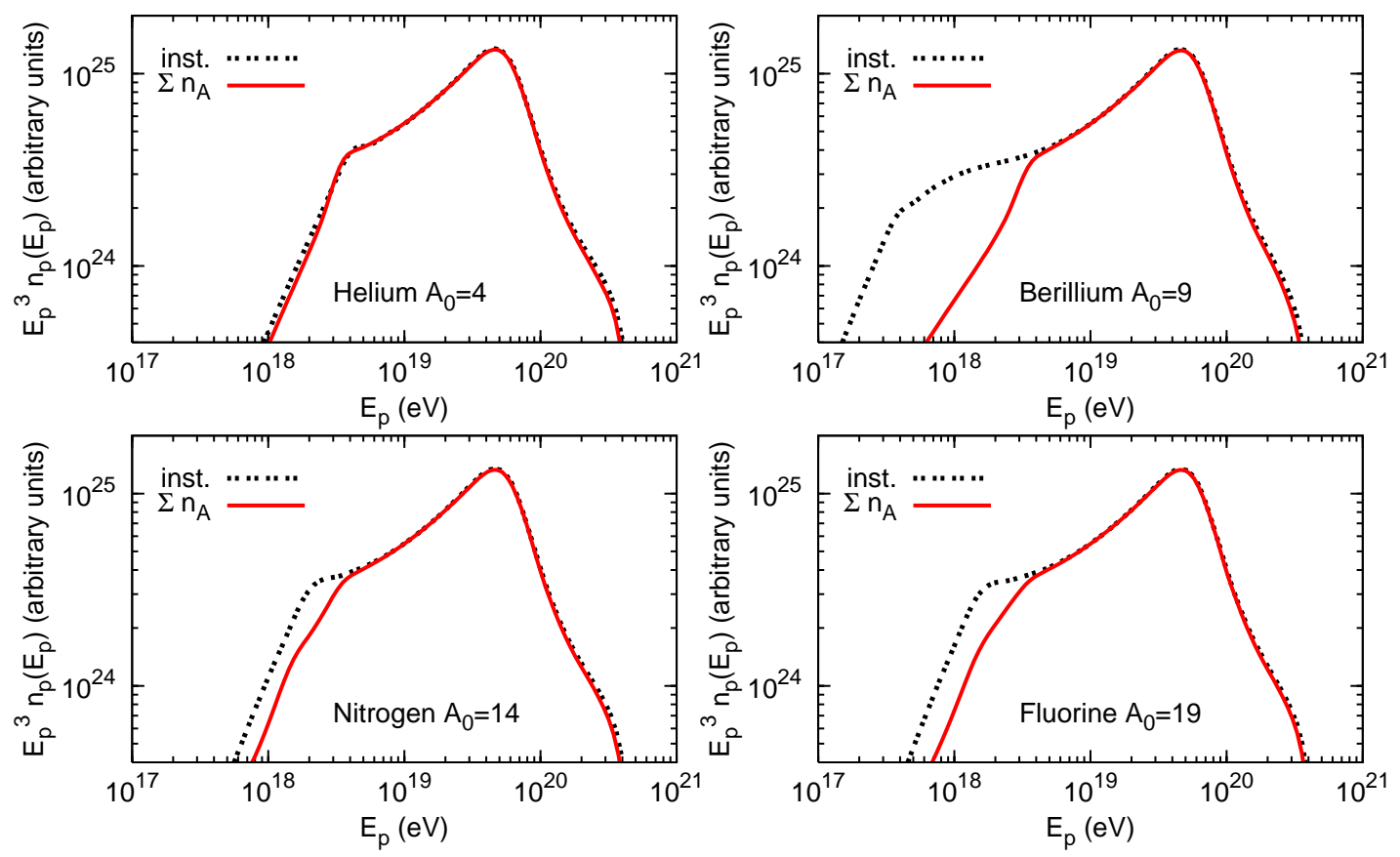

Figure 12: Spectra of secondary protons computed by the A-intermediate method (continuous red curves) and in the instantaneous approximation (black dotted curves) for different $A_{0}$. The generation spectral index is $\gamma_{g}=2.3$ in all cases.
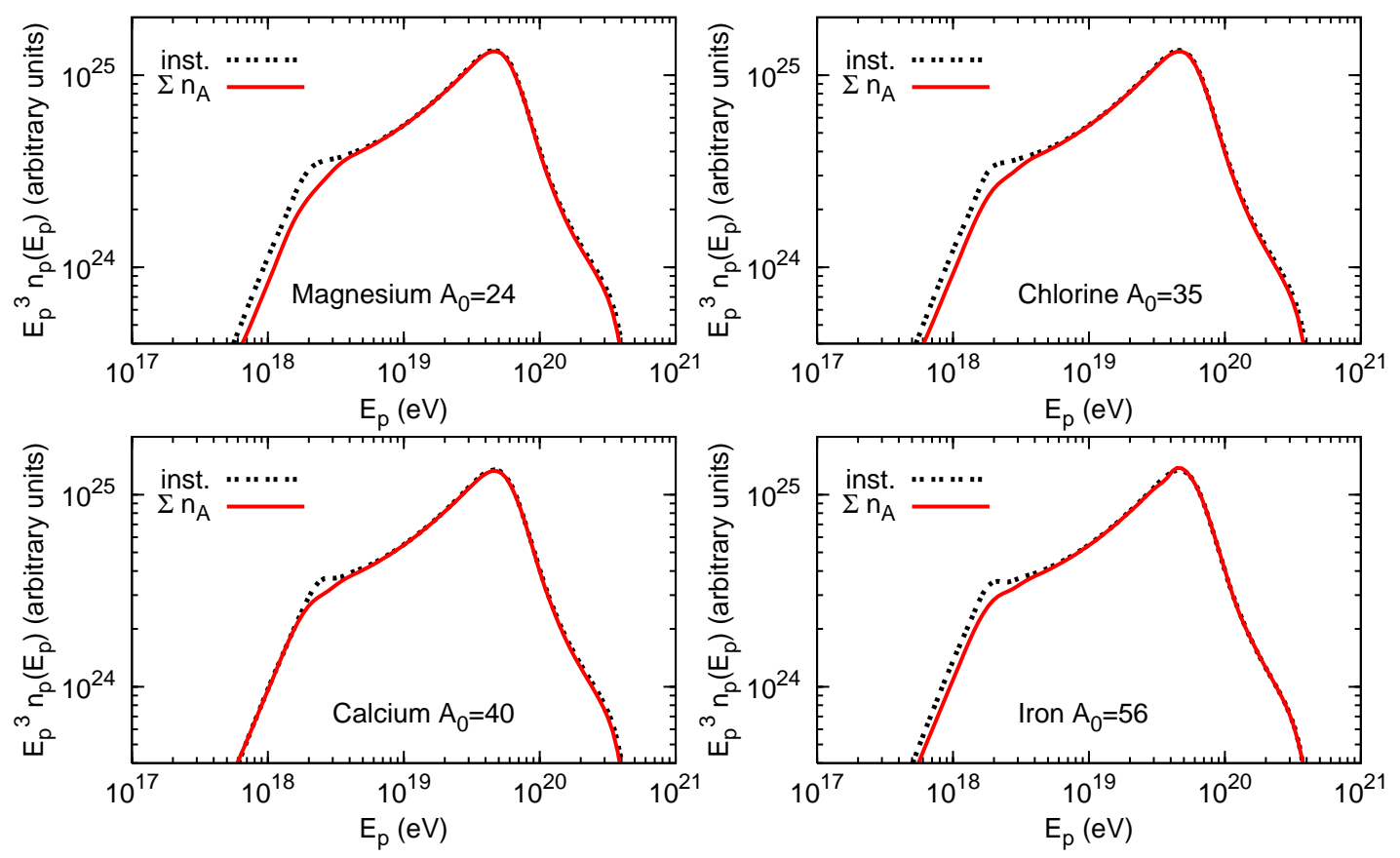

Figure 13: The same as in Fig. 12 for heavy nuclei. 
$z_{A} \approx z_{g}$ and $\Gamma_{A} \approx \Gamma_{g}$ in Eq. (28), one obtains after simple calculations

$$
n_{p}^{A}\left(\Gamma_{p}\right)=\frac{\gamma_{g}-2}{A_{0} m_{N}} \frac{\mathcal{L}_{0}}{H_{0}} \int_{z_{g}^{\min }}^{z_{g}^{\max }} d z_{g} \frac{\left[\Gamma_{g}^{p}\left(\Gamma_{p}, z_{g}\right)\right]^{-\gamma_{g}}}{\left(1+z_{g}\right) \sqrt{\Omega_{m}\left(1+z_{g}\right)^{3}+\Omega_{\Lambda}}} \frac{d \Gamma_{g}^{p}}{d \Gamma_{p}} .
$$

The total flux of protons is obtained by summation in Eq. (32) over all $A$. Since limits of integration depend weakly on $A$, it means multiplication of Eq. (32) to $A_{0}$, which results in instantaneous flux given by Eq. (31).

We shall discuss now the calculated spectra presented in Figs. 12 and 13 .

One may observe that agreement between the exact ( $A$-intermediate) and instantaneous method of calculations is precise at high energies $\left(\Gamma>\Gamma_{c}\right)$ and approximate at low energies $\left(\left(\Gamma<\Gamma_{c}\right)\right.$ and $\left.\left(\Gamma \ll \Gamma_{c}\right)\right)$, as it is expected.

For small $A$ the excellent agreement at low energies is observed for ${ }^{4} \mathrm{He}$. This is an exceptional case: the energy threshold of photo-disintegration for ${ }^{4} \mathrm{He}$ is much higher than for its daughter nuclei: ${ }^{3} \mathrm{He}$ and D. Hence, when ${ }^{4} \mathrm{He}$ starts to photo-disintegrate, its daughter-nuclei disintegrate too. Based on this argument we expect that in the Helium case the spectra of secondary protons calculated by both methods should be identical at all Lorentz factors, including the low ones, and this expectation is confirmed by the upper-left panel of Fig. 12.

We now come over to the "Beryllium excess" in the spectrum of the instantaneous decay, shown in the upper-right panel of Fig. 12. The nature of this effect is simple: $\Gamma_{c}^{\mathrm{Be}} \ll \Gamma_{c}^{\mathrm{He}}$ and since ${ }^{4} \mathrm{He}$ is born with Lorentz factor of ${ }^{9} \mathrm{Be}$, the former can be stable and secondary protons are not produced. For $\Gamma>\Gamma_{c}^{\mathrm{Be}}$ it gives a suppression of the secondary proton flux. Under the assumption of an instantaneous decay this effect is absent.

Beryllium effect works for all $A_{0}>9$ and since it affects only 8 protons from two ${ }^{4} \mathrm{He}$ nuclei, its influence on heavy nuclei is weaker, as one observes from Fig. 13.

The shape of the proton spectra in Figs. 12 and 13 can be naturally explained. They have two spectral breaks: the high energy steepening, which is the usual GZK cutoff at energy $E \sim 5 \times 10^{19} \mathrm{eV}$, and the low-energy steepening which coincides with $\Gamma_{c}: \sim 4 \times 10^{9}$ for ${ }^{4} \mathrm{He}, \sim 4 \times 10^{8}$ for ${ }^{9} \mathrm{Be}$ etc). Below $\Gamma_{c}$ the adiabatic energy losses dominate and it explains the flat spectrum $n_{p}\left(E_{p}\right)$ there, i.e. steep $E^{3} n_{p}(E)$ spectrum in Figs. 12 and 13 , It is different from $\propto E^{-\gamma_{g}}$ because $z_{\min }$ in Eq. (28) and Eq. (31) depends on $\Gamma$, i.e. on $E_{p}$.

We complete this section with a remark about the universality of the secondary-proton spectrum. Using Eq. (31) for the instantaneous spectrum we proved its universality. i.e. independence of $A_{0}$. The intermediate $\mathrm{A}$ spectrum has the same property, as it was proved above. In other words the normalised spectrum calculated for one $A_{0}$ is valid for any other $A_{0}$ with the same normalization.

\subsection{Primary nuclei}

We calculate now the diffuse flux of primary nuclei $A_{0}$, which arrive undestroyed from the sources. The comoving space density of these nuclei $n_{A_{0}}(\Gamma, t)$ is described by the kinetic equation

$$
\frac{\partial n_{A_{0}}(\Gamma, t)}{\partial t}-\frac{\partial}{\partial \Gamma}\left[b_{A_{0}}(\Gamma, t) n_{A_{0}}(\Gamma, t)\right]+\frac{n_{A_{0}}(\Gamma, t)}{\tau_{A_{0}}(\Gamma, t)}=Q_{A_{0}}(\Gamma, t),
$$

where $Q_{A_{0}}(\Gamma, t)$ is the generation rate of primary nuclei per unit time and comoving volume, and $\tau_{A_{0}}(\Gamma, t)$ is the photo-disintegration lifetime of the nucleus $A_{0}$ calculated as $\left[A_{0} \beta_{\text {dis }}\left(A_{0}, \Gamma, z\right)\right]^{-1}$ with $\beta_{\text {dis }}$ given by Eq. (7). 

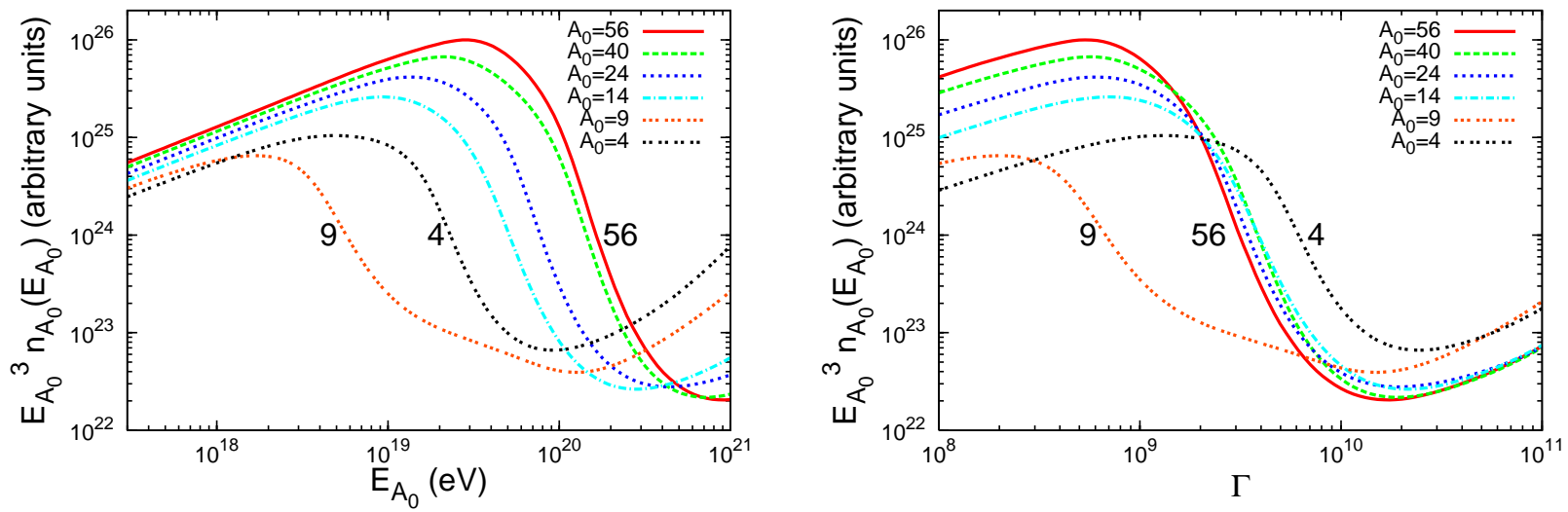

Figure 14: Spectrum of primary nuclei with various $A_{0}$ as function of energy (left panel) and Lorentz factor (right panel).

As found in appendix $\mathrm{C}$ the solution of the kinetic equation (33) reads

$$
n_{A_{0}}(\Gamma)=\int_{0}^{\infty} d z_{g}\left|\frac{d t_{g}}{d z_{g}}\right| Q_{A_{0}}\left(\Gamma_{g}, z_{g}\right) \frac{d \Gamma_{g}}{d \Gamma} e^{-\eta\left(\Gamma_{g}, z_{g}\right)}
$$

where $n_{A_{0}}(\Gamma)$ is the space density of nuclei $A_{0}$ at $z=0, \Gamma_{g}=\mathcal{G}\left(A_{0}, \Gamma, z_{0}=0, z_{g}\right)$ is the Lorentz factor of nuclei $A_{0}$ at the moment of generation $z_{g}$ calculated by the evolution trajectory, with fixed $A_{0}$, which starts from $\Gamma$ at $z_{0}=0$. The ratio $d \Gamma_{g} / d \Gamma$ is also calculated with fixed $A_{0}$. The quantity $\eta$ takes into account the photo-disintegration of the propagating nucleus:

$$
\eta\left(\Gamma_{g}, z_{g}\right)=\int_{t\left(z_{g}\right)}^{t_{0}} \frac{d t}{\tau_{A_{0}}(\Gamma(t), t)} .
$$

The lower limit of integration in Eq. (34) reflects the assumption of an homogeneous distribution of the sources. The upper limit is imposed by the factor $\exp (-\eta)$ accompanied by the condition of a maximum acceleration Lorentz factor: $Q_{A_{0}}\left(\Gamma_{g}\right)=0$, if $\Gamma_{g} \geq \Gamma_{\max }^{\mathrm{acc}}$.

In Fig. 14 we plot the flux of primary nuclei for various $A_{0}$, using the injection spectrum with $\gamma_{g}=2.3$. The steepening of the spectra in Fig. 14 starts from the Lorentz factor where pair-production energy losses are equal to adiabatic energy losses. Comparison of Figs. 2 and 14 confirms it. It is worth noting that this criterion for the beginning of the steepening was obtained first in [39].

\section{Coupled kinetic equations (CKE)}

In this section we present a straightforward method of calculation for UHE nuclei fluxes, based on the analytic solution of a complete set of coupled kinetic equations. The great advantage of this method is a simple expression for the generation rates of secondary nuclei and protons, which do not include integration with independently determined lower and upper limits. We consider as before the expanding universe filled homogeneously by accelerated primary and secondary nuclei, including the secondary protons. As discussed in section 2.1, these secondary particles are produced mainly by one-nucleon photo-disintegration process $(A+1)+\gamma \rightarrow A+p$. In this approximation one can write 
the generation rate for any secondary nucleus $A$ as a function only of the equilibrium distribution of the parent nucleus $(A+1)$, namely:

$$
Q_{A}(\Gamma, z)=\frac{n_{A+1}(\Gamma, z)}{\tau_{A+1}(\Gamma, z)}
$$

where $\tau_{A}(\Gamma, z)$ is given by Eq. (12) and (77). Taking it into account one may write a set of coupled kinetic equations which describes the equilbrium of primary nuclei $A_{0}$ with the products of their decay

$$
\begin{aligned}
\frac{\partial n_{A_{0}}(\Gamma, t)}{\partial t}-\frac{\partial}{\partial \Gamma}\left[n_{A_{0}}(\Gamma, t) b_{A_{0}}(\Gamma, t)\right]+\frac{n_{A_{0}}(\Gamma, t)}{\tau_{A_{0}}(\Gamma, t)} & =Q_{A_{0}}(\Gamma, t) \\
\frac{\partial n_{A_{0}-1}(\Gamma, t)}{\partial t}-\frac{\partial}{\partial \Gamma}\left[n_{A_{0}-1}(\Gamma, t) b_{A_{0}-1}(\Gamma, t)\right]+\frac{n_{A_{0}-1}(\Gamma, t)}{\tau_{A_{0}-1}(\Gamma, t)} & =\frac{n_{A_{0}}(\Gamma, t)}{\tau_{A_{0}}(\Gamma, t)} \\
& \vdots \\
\frac{\partial n_{A}(\Gamma, t)}{\partial t}-\frac{\partial}{\partial \Gamma}\left[n_{A}(\Gamma, t) b_{A}(\Gamma, t)\right]+\frac{n_{A}(\Gamma, t)}{\tau_{A}(\Gamma, t)} & =\frac{n_{A+1}(\Gamma, t)}{\tau_{A+1}(\Gamma, t)}
\end{aligned}
$$

The solution of the kinetic equation for primaries $A_{0}$ is given by

$$
n_{A_{0}}(\Gamma, z)=\int_{z}^{z_{\max }} \frac{d z^{\prime}}{\left(1+z^{\prime}\right) H\left(z^{\prime}\right)} Q_{A_{0}}\left(\Gamma^{\prime}, z^{\prime}\right) \frac{d \Gamma^{\prime}}{d \Gamma} e^{-\eta_{A_{0}}\left(\Gamma^{\prime}, z^{\prime}\right)}
$$

with

$$
\eta_{A_{0}}\left(\Gamma^{\prime}, z^{\prime}\right)=\int_{z}^{z^{\prime}} \frac{d z^{\prime \prime}}{\left(1+z^{\prime \prime}\right) H\left(z^{\prime \prime}\right)} \frac{1}{\tau_{A_{0}}\left(\Gamma^{\prime \prime}, z^{\prime \prime}\right)}
$$

and the solution for an arbitrary secondary nuclei $A$ is

$$
n_{A}(\Gamma, z)=\int_{z}^{z_{\max }} \frac{d z^{\prime}}{\left(1+z^{\prime}\right) H\left(z^{\prime}\right)} \frac{n_{A+1}\left(\Gamma^{\prime}, z^{\prime}\right)}{\tau_{A+1}\left(\Gamma^{\prime}, z^{\prime}\right)} \frac{d \Gamma^{\prime}}{d \Gamma} e^{-\eta_{A}\left(\Gamma^{\prime}, z^{\prime}\right)}
$$

with

$$
\eta_{A}\left(\Gamma^{\prime}, z^{\prime}\right)=\int_{z}^{z^{\prime}} \frac{d z^{\prime \prime}}{\left(1+z^{\prime \prime}\right) H\left(z^{\prime \prime}\right)} \frac{1}{\tau_{A}\left(\Gamma^{\prime \prime}, z^{\prime \prime}\right)} .
$$

In each of these equations we assume $A=$ const and the Lorentz factor changing due to adiabatic and pair-production energy losses. Accordingly, the ratio $d \Gamma^{\prime} / d \Gamma$ is given by

$$
\frac{d \Gamma^{\prime}}{d \Gamma}=\frac{1+z^{\prime}}{1+z} \exp \left[\frac{Z^{2}}{A} \int_{z}^{z^{\prime}} \frac{\left(1+z^{\prime \prime}\right)^{2} d z^{\prime \prime}}{H\left(z^{\prime \prime}\right)}\left(\frac{d b_{0}^{p}(\tilde{\Gamma})}{d \tilde{\Gamma}}\right)_{\tilde{\Gamma}=\left(1+z^{\prime \prime}\right) \Gamma^{\prime \prime}}\right]
$$

where $b_{0}^{p}(\Gamma)=d \Gamma / d t$ is the Lorentz-factor loss per unit time for protons at $z=0$ due to pair production.

The procedure to solve the system of equations (37) consists in finding the analytic solution $n_{A_{0}}(\Gamma, t)$ for the first equation in the form (38), and putting it into the second equation. Taking the solution of this second equation, given by Eq. (40) with $A=A_{0}-1$, one finds $n_{A_{0}-1}(\Gamma, t)$. Continuing this procedure one obtains the space density (fluxes) for all secondary nuclei $n_{A}(\Gamma, t)$. 
Note, that in this method there is no problem with the limits of integration: the lower limit is $z$ by definition and the upper limit is regulated by the factor $e^{-\eta}$, while $z_{\max }$ here plays only a symbolic role. The generation rate $Q_{A}(\Gamma, z)$ given by Eq. (36) tends to zero at small $\Gamma$ due to factor $\tau_{A+1} \rightarrow \infty$ at small Lorentz factors.

For the calculations of secondary protons spectrum we use the $A$-associating proton approach developed in section 3.2 . The secondary nucleons $N$ which accompany production of $A$-nuclei in the process $(A+1) \rightarrow A+N$ have the same generation rate

$$
Q_{p}^{A}(\Gamma, z)=Q_{A}(\Gamma, z)=\frac{n_{A+1}(\Gamma, z)}{\tau_{A+1}(\Gamma, z)} .
$$

The kinetic equation for secondary protons is given by Eq. (25) with the solution described by Eq. (26). Using the generation rate in the form of Eq. (43) this solution reads

$$
n_{p}^{A}(\Gamma, z)=\int_{z}^{z_{\max }} \frac{d z^{\prime}}{\left(1+z^{\prime}\right) H\left(z^{\prime}\right)} \frac{n_{A+1}\left(\Gamma^{\prime}, z^{\prime}\right)}{\tau_{A+1}\left(\Gamma^{\prime}, z^{\prime}\right)} \frac{d \Gamma^{\prime}\left(z^{\prime}\right)}{d \Gamma},
$$

where $\Gamma^{\prime}\left(z^{\prime}\right)=G_{p}\left(\Gamma, z, z^{\prime}\right)$ is the proton trajectory, and Eq. (44) is valid for any arbitrary initial $z$ including $z=0$. In fact, the actual lower limit of integration $z_{\text {min }}$ is automatically provided by $\tau_{A+1}\left(\Gamma^{\prime}, z^{\prime}\right)$, and this gives a great advantage of the CKE method in comparison with the combined method, where $z_{\min }$ is calculated independently, using some particular features of the trajectory evolution. As will be seen by the example of secondary $A$-nuclei, the brothers of secondary protons, the structure of the lower-limit cutoff exposed by Eq. (52) is quite different from the sharp cutoff at $z_{\text {min }}$ introduced in the combined method.

The upper limit of integration $z_{\max }$ in Eq. (44) is quite different from secondary nuclei, where it is imposed by the life-time $\tau_{A}$ in the form of $e^{-\eta_{A}}$. In the case of secondary protons the upper limit is provided by $\Gamma_{\max }$, which the brother $A$-nucleus (or parent $A+1$ nucleus) is allowed to have. Since at these very large Lorentz factors the approximation of explosive trajectories is fully justified, we can use $\Gamma_{\max }$ for primary nucleus $A_{0}$ and $z_{\max }$ is given (in case of initial $z=0$ ) by the equation for the proton trajectory $\Gamma_{\max }=G_{p}\left(\Gamma, 0, z_{\max }\right)$, where $\Gamma$ is the proton Lorentz factor at $z=0$.

As we discuss below, the fluxes obtained by the CKE method for secondary nuclei and protons differ, most notably at low energies, from that in the combined method (see section (3). This difference is due to the lower limit of integration in the Eq. (40) and (44). In the combined method it is sharply fixed at $z_{\min }$ as discussed in section 3.1, while in the CKE method the integrand is automatically suppressed by the term $\tau_{A+1}\left(\Gamma^{\prime}, z^{\prime}\right)$ (see discussion below).

The fluxes of secondary nuclei and protons are displayed in Fig. 15.

We will discuss now the solutions obtained for secondary nuclei and protons. In particular we will study these solutions in their analytic form to compare them with the combined method.

First we obtain the analytic solutions of Eqs. (37) in the high-energy asymptotic regime, when all $\tau_{A}$ are very short. We re-write the solution (38) in terms of the cosmological time using for $e^{-\eta}$ the expression of Eq. (76) (see appendix C):

$$
n_{A_{0}}(\Gamma, t)=\int_{t_{g}}^{t} d t^{\prime} Q_{A_{0}}\left(\Gamma^{\prime}, t^{\prime}\right) \frac{d \Gamma^{\prime}}{d \Gamma} \exp \left[-\int_{t^{\prime}}^{t} \frac{d t^{\prime \prime}}{\tau_{A_{0}}\left(\Gamma^{\prime \prime}, t^{\prime \prime}\right)}\right] .
$$

Introducing the propagation time $t_{\text {prop }}=t-t^{\prime}$ as integration variable in Eq. (45) and taking $Q_{A_{0}} d \Gamma^{\prime} / d \Gamma$ out of the integral as a slowly variable quantity, one obtains after simple 

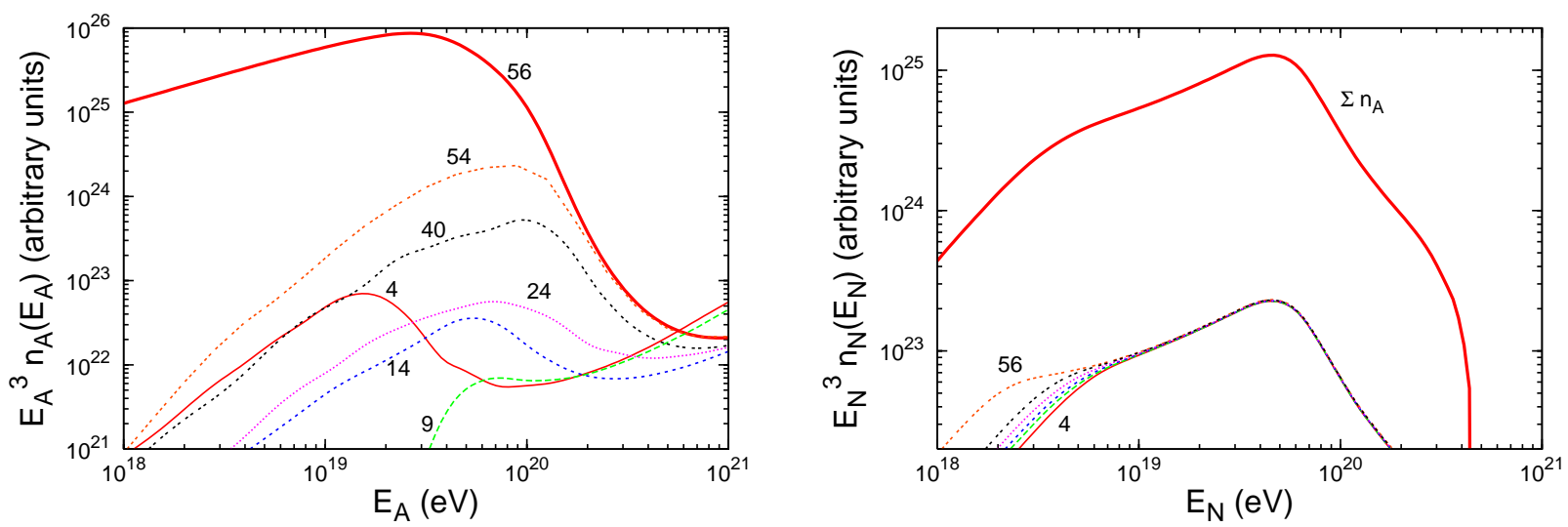

Figure 15: Flux of secondary nuclei (the primary $A_{0}=56$ included) (left panel) and of the secondary protons (right panel) as function of energy. The numbers on the curves show A. $\Sigma_{A}$ is related with the total flux of protons produced by the primaries $A_{0}=56$, the numbers indicate fluxes for each $A$-associating proton. The discussion of these results and comparison with the combined method is given in the next section.

calculations

$$
n_{A_{0}}(\Gamma, t)=Q_{A_{0}}(\Gamma, t) \tau_{A_{0}}(\Gamma, t)
$$

and

$$
Q_{A_{0}-1}(\Gamma, t)=n_{A_{0}}(\Gamma, t) / \tau_{A_{0}}(\Gamma, t)=Q_{A_{0}}(\Gamma, t) .
$$

Repeating these calculations for $A<A_{0}$ we obtain for the high-energy asymptotic regime

$$
Q_{A}(\Gamma, t)=Q_{A+1}(\Gamma, t)=\ldots=Q_{A_{0}}(\Gamma, t)
$$

and

$$
n_{A}(\Gamma, t)=Q_{A}(\Gamma, t) \tau_{A}(\Gamma, t)=Q_{A_{0}}(\Gamma, t) \tau_{A}(\Gamma, t),
$$

i.e. the same high-energy regime that we obtained in Eqs. (23) and (24) in the combined method. In particular, the equality (48) is the same obtained in the combined method for the explosive regime, which is provided by short $\tau_{A}$ like in the case above.

We will now come over to the general case valid also for low $\Gamma$. To obtain the general solution $n_{A}(\Gamma, t)$ in the analytic form we put into Eq. (40) the value $n_{A+1}\left(\Gamma^{\prime}, z^{\prime}\right)$ determined from the preceding equation

$$
n_{A+1}\left(\Gamma^{\prime}, z^{\prime}\right)=\int_{z^{\prime}}^{z_{\max }} \frac{d z^{\prime \prime}}{\left(1+z^{\prime \prime}\right) H\left(z^{\prime \prime}\right)} \frac{n_{A+2}\left(\Gamma^{\prime \prime}, z^{\prime \prime}\right)}{\tau_{A+2}\left(\Gamma^{\prime \prime}, z^{\prime \prime}\right)} \frac{d \Gamma^{\prime \prime}}{d \Gamma^{\prime}} e^{-\eta_{A}\left(\Gamma^{\prime \prime}, z^{\prime \prime}\right)} .
$$

Repeating this procedure with increasing $A$ until we reach $A_{0}$, we obtain the general expression with many-fold integral

$n_{A}(\Gamma, z)=\left[\prod_{i} \int_{z_{i}}^{z_{\max }} d z_{i} \frac{e^{-\eta_{A_{0}-i}\left(\Gamma_{i}, z_{i}\right)}}{\left(1+z_{i}\right) H\left(z_{i}\right) \tau_{A_{0}+1-i}\left(\Gamma_{i}, z_{i}\right)}\right] \int_{z_{1}}^{z_{\max }} d z^{\prime} \frac{Q_{A_{0}}\left(\Gamma^{\prime}, z^{\prime}\right)}{\left(1+z^{\prime}\right) H\left(z^{\prime}\right)} \frac{d \Gamma^{\prime}}{d \Gamma} e^{-\eta_{A_{0}}\left(\Gamma^{\prime}, z^{\prime}\right)}$

where $d \Gamma^{\prime} / d \Gamma$ is taken along the $A$-variable trajectory. Using Eq. (15) for $Q_{A_{0}}\left(\Gamma^{\prime}, z^{\prime}\right)$ and assuming that the external integration results in some functions $f(\Gamma, z)$ (we calculated 
these functions numerically) we obtain

$$
n_{A}(\Gamma, 0)=\int_{0}^{z_{\max }} d z f(z) \int_{z}^{z_{\max }} \frac{d z^{\prime}}{\left(1+z^{\prime}\right) H\left(z^{\prime}\right)} \mathcal{L}_{0} \frac{\gamma_{g}-2}{A_{0} m_{N}}\left[\Gamma_{g}\left(\Gamma, A, z^{\prime}\right)\right]^{-\gamma_{g}} \frac{d \Gamma_{g}}{d \Gamma} e^{-\eta_{A_{0}}\left(\Gamma_{g}, z^{\prime}\right)},
$$

with $f(z)$ rapidly vanishing when $z \rightarrow 0$ and $z \rightarrow z_{\max }$.

The presentation of our solution as a manyfold integral allows us to discuss whether one should classify this solution as analytic or implicit-analytic, as in the first paper of [46] is formulated. We follow the definition of the analytic solution used in many monographs and text books: analytic solution is the one which can be presented by a finite number of quadratures, or a solution which has the form of a finite number of successive integrations. With these definitions Eqs. (51) and (52) demonstrate that the solutions we obtain are analytic.

Comparison of the solutions (51) and (52) with $n_{A}(\Gamma, 0)$ given by Eq. (22) for the combined method shows similarity and differences. The crucial one consists in $z_{\text {min }}$. While in the combined method this is a well determined function of $\Gamma$, in the CKE method the lower limit is given by different values of $z$ weighted by the function $f(z)$.

Numerical calculations show a very good agreement between the two methods at the highest energies and for all primary nuclei $A_{0}$ at all energies. For secondary protons the disagreement is moderate, but for the secondary nuclei there is a strong disagreement at low energies. We see the main reason for this disagreement in the $z_{\min }(\Gamma)$ calculations. In the combined model $z_{\text {min }}$ is calculated from reasonable physics connected with trajectories. In the CKE method the lower limit appears automatically, without any assumption, directly from the kinetic equations. We think that as far as numerical results are concerned, this last method should be trusted more.

\section{Comparison with other computation schemes}

In this section we will compare the CKE spectra with those present in literature and obtained by different methods. In paper II we will present such comparison in a more detailed way taking into account the total background radiation, given by the sum of EBL and CMB, here we will restrict our study to the case of CMB only. This comparison cannot be done straightforwardly, because in all available calculations EBL and CMB are not used separately.

We solve this problem introducing three criterions of agreement.

In Figs [16 taken from paper II the photo-disintegration lifetimes $\tau_{A}$ are given for EBL and CMB separately (paper II and references therein). For convenience we present these graphs here in Fig. 16 for Iron (right panel) and Nitrogen (left panel). One can see that lifetimes $\tau_{A}$ for EBL and CMB are merged sharply at the critical Lorentz factor $\Gamma_{m}$ equal to $3 \times 10^{9}$ and $4 \times 10^{9}$ for Iron and Nitrogen, respectively; the corresponding energies are $1.7 \times 10^{20} \mathrm{eV}$ for $\mathrm{Fe}$ and $5.6 \times 10^{19} \mathrm{eV}$ for N. Therefore, the first criterion of agreement between spectra for EBL+CMB (literature) and CMB only (our calculations) is given by the merging energy $E_{m}=A \Gamma_{m} m_{N}$ taken from Fig. 16. The second criterion is the agreement of the spectra with CMB only and with EBL+CMB above the merging energy, because at these energies photo-disintegration on CMB strongly dominates.

The third criterion of agreement is given by the secondary proton spectrum. EBL produces secondary protons with Lorentz-factors below $\Gamma_{m}$, i.e. with low energies $E_{p} \lesssim$ $\Gamma_{m} m_{N} \sim 3 \times 10^{18} \mathrm{eV}$. Protons with higher energies are produced on CMB only. Therefore, 

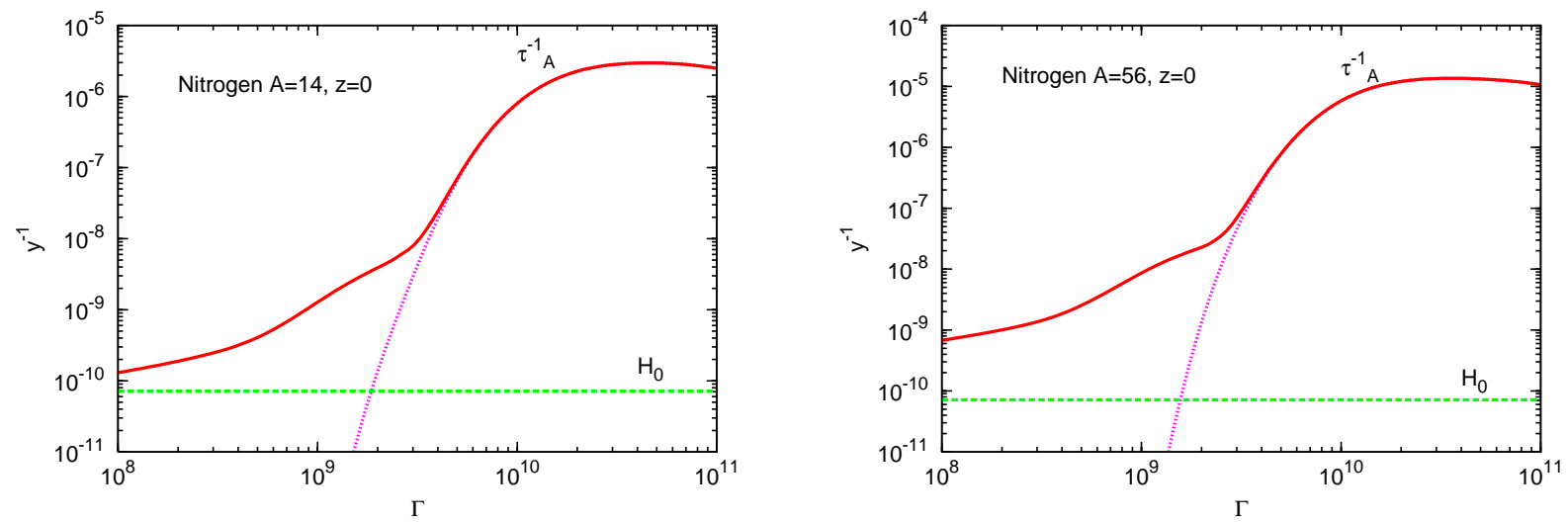

Figure 16: Inverse photo-disintegration lifetime $\tau_{A}^{-1}$ for Nitrogen (left panel) and Iron (right panel) nuclei for CMB background only (dotted magenta curve) and for CMB+EBL (continuos red curve). The merging Lorentz-factor is defined as intersection of these two curves.

the agreement of secondary protons spectra on EBL+CMB (literature) and on CMB only (our calculations) at $E_{p} \gtrsim \Gamma_{m} m_{N}$ gives one more proof of the discussed agreement.

Let us now come over to the explicit comparison of our spectra with those in literature. The most interesting case for us is the comparison with the results of [44, 45], where the same kinetic equations are used. The essential difference is that in our work the kinetic equations are solved analytically, while in [44, 45] solutions are obtained numerically. Thus this comparison can be considered as a numerical test of the CKE method.

In the left panel of Fig. 17 we show the comparison of our analytic calculations (using only CMB) with the numerical solution of the kinetic-equation as calculated in [44], where EBL is also included. The parameters used in these calculations are: $\gamma_{g}=2.2$ and $E_{\text {max }}=6.4 \times 10^{20} Z_{0} \mathrm{eV}$, where $Z_{0}$ is the atomic charge number of the injected particles. Computations are performed assuming a homogeneous distribution of sources with a pure Iron injection.

The full red line shows the all-particles spectrum in our work (CMB only) and red asterisks show the spectrum [44] produced on EBL+CMB. One may notice the suppression of latter spectrum by photo-disintegration on EBL. These two spectra merge at $1.5 \times$ $10^{20} \mathrm{eV}$, as expected. The secondary protons spectrum shown by green dashed line (our calculations) and by green asterisks [44] coincide well too.

We will compare now our spectra with MC simulation using the computation by Allard et al [58]. In this work the accelerated particles are assumed to be Iron nuclei, the generation index is $\gamma_{g}=2.3$, the sources are assumed to be homogeneously distributed in space, and the maximum energy of acceleration is $E_{\max }=2 \times 10^{20} Z_{0} \mathrm{eV}$. The results are shown in the right panel of Fig. 17, where the computation by Allard et al [58] includes EBL and CMB, while our calculations - only CMB. All three criterions for a good agreement are present in these two spectra: the merging energy is in the right place, both spectra coincide above the merging energy and secondary-protons spectrum agree at all energies.

It is interesting to comment on the agreement between all-particles spectra in both left and right panels of Fig. 17. The agreement above the merging energy is trivial and is explained by the dominance of CMB photons in the formation of the highest energy part of the spectrum. Agreement of spectra at low energies, where EBL photons 

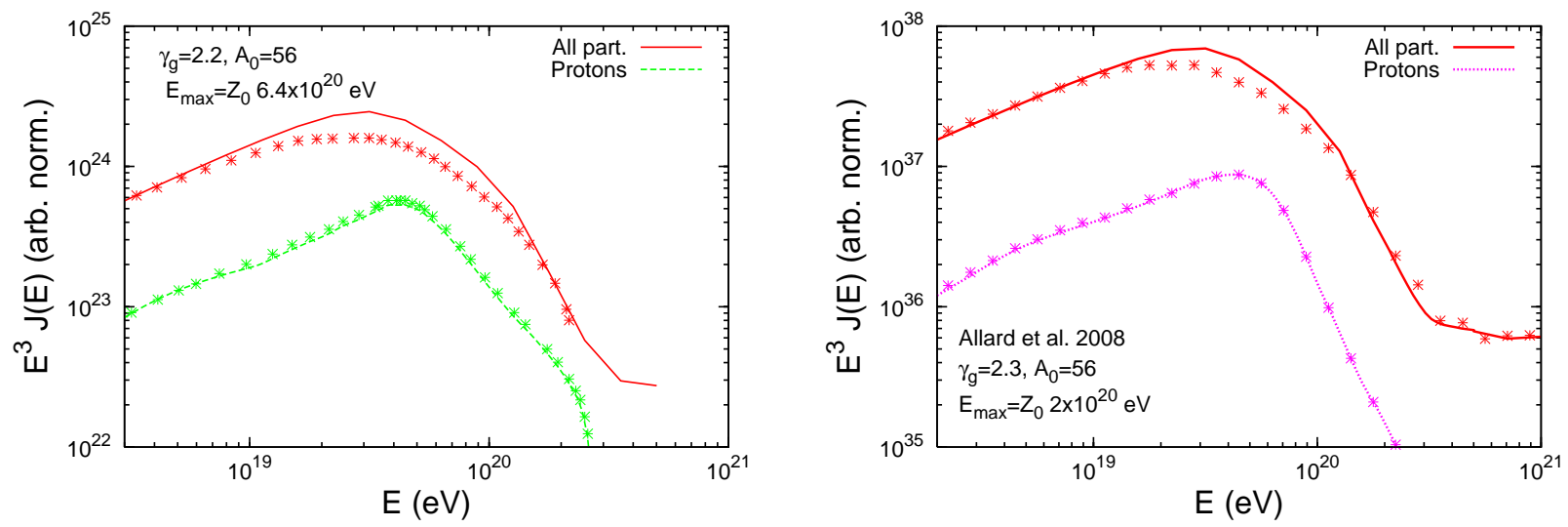

Figure 17: Left panel:Comparison of energy spectra with CMB only (our calculations) with [44], where both EBL and CMB are included. Primaries at the source are Iron nuclei. Solid red line shows all-particles spectrum from our calculations, the red asterisks present all-particles spectrum from [44]. One may see the EBL suppression of the latter flux. Both fluxes merge at $1.5 \times 10^{20} \mathrm{eV}$ as expected. The secondary-proton spectra in the lower part of the figure coincide too. See the text for more details. Right panel: Comparison of spectra with CMB only (our calculations) with MC simulation by Allard et al [58] which includes EBL and CMB. In the upper part of the figure the all-particles spectra are compared, in the lower part - that of secondary protons. The solid lines show our calculations and asterisks - calculations by Allard et al.

dominate, is caused by an approximate conservation of the number of nuclei: in the process $A+\gamma \rightarrow(A-1)+N$ the nucleon has energy A times lower than nucleus, thus it has an energy out of the range we considered here. The suppression of the nuclei flux appears at the intermediate energies due to the full destruction of nuclei by EBL photons.

We can conclude stating that the analysis performed shows a very good agreement of the fluxes computed with the three methods analyzed taking into account only the CMB, when the flux of target photons is rigidly fixed.

To conclude this section we will briefly discuss the comparison of our theoretical results with the Auger observations. The attempt to compare the spectra obtained in this paper through a theoretical toy-model, based on the simplified assumptions of a pure Iron injection and only CMB photons as target, with the observed spectrum may be motivated as follows.

In the rigidity-acceleration models with $E_{\max } \propto Z$ the end of the spectrum is dominated by the heaviest nuclei, like e.g. in the disappointing model [32. Moreover, CMB photons dominate the photo-disintegration process only above the merging energy, $E_{m} \approx 1.7 \times 10^{20} \mathrm{eV}$ for Iron. However, due to the approximate conservation of the number of nuclei, the EBL suppression of the all-particles spectrum is not strong (see Fig. 17). Therefore, it can be expected that our simplified model gives a good description of he Auger spectrum at the highest energies. The comparison is given in Fig. 18 with the computed all-particles spectrum shown by the solid line.

To conclude, we want to emphasize again that Fig. 18 has to be considered only as an illustration, the aim of the present paper is to introduce, on theoretical grounds, new techniques to calculate analytically the UHE nuclei fluxes. The application of the 


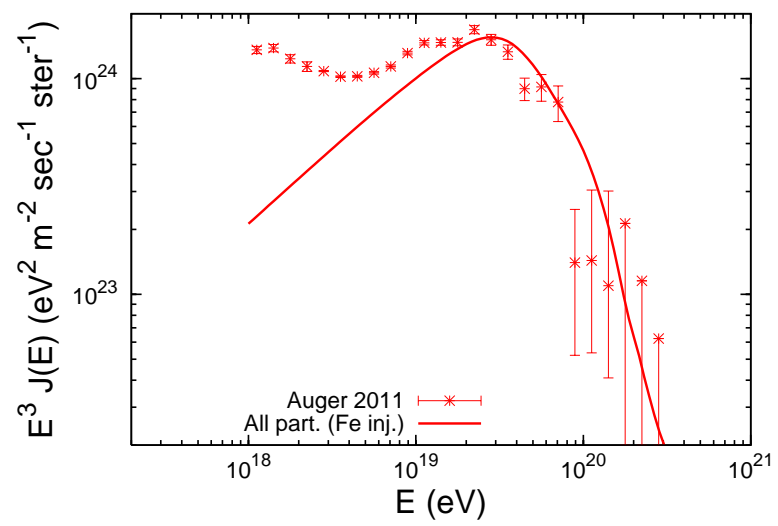

Figure 18: Comparison of the Auger 2011 energy spectrum [10] with the all-particles spectrum computed in the present paper with $\gamma_{g}=2.3 E_{\text {max }}=Z_{0} 2 \times 10^{20} \mathrm{eV}$ and a pure Iron injection at the sources.

technique developed here in detailed calculations of the spectra with a coherent comparison with experimental data will be presented in a forthcoming paper.

Finally, we do not discuss here the comparison with HiRes and Telescope Array observations because they show a strongly proton-dominated mass composition [24, 18, therefore cannot be described in the framework of a pure iron injection used in this paper.

\section{Discussion and Conclusions}

In this paper we have studied comparatively three analytic methods to determine the diffuse spectra of ultra-high energy nuclei propagating through background radiations: (i) trajectory method, (ii) kinetic-equation method combined with trajectory calculations (the combined method), and (iii) coupled kinetic equations (CKE).

We summarize first our general approach.

We have calculated the diffuse spectra of primary and secondary nuclei, and secondary protons in the case of homogeneously distributed sources. According to the propagation theorem [63] in this case the spectra do not depend on the specific way of propagation, and thus one may use rectilinear propagation, or not thinking about space propagation at all, imaging all secondary nuclei and protons filling the space homogeneously together with primary nuclei.

Evolution in time is the basic feature of our problem. A primary nucleus $A_{0}$ is born due to acceleration and, interacting with the background radiation, give rise to the secondary nuclei and protons.

With the help of two coupled differential equations for the propagation of a primary nucleus in the backward time, i.e. increasing redshift, we found the evolution trajectories $A(z)=\mathcal{A}\left(A, \Gamma, z_{0}, z\right)$ and $\Gamma(z)=\mathcal{G}\left(A, \Gamma, z_{0}, z\right)$, where the first three arguments fix the initial conditions. In the evolution equations the atomic number $A$ changes continuously with $z$, but we use the continuous trajectory $A(z)$ only to determine the integer $A$ positions on the trajectory: $A, A+1$ etc. At these points we assume an instantaneous photodisintegration, e.g. $(A+1)+\gamma_{\mathrm{CMB}} \rightarrow A+N$. The Lorentz factor between the points of photo-disintegration is calculated assuming $A=$ const. The trajectories are basically 
needed to compute the generation parameters $z_{g}$ and $\Gamma_{g}$, at which $A(z)$ reaches $A_{0}$. Apropos, the formal solution of the coupled trajectory equations (9) with continuous $A$ results in the same $\Gamma_{g}$ and $z_{g}$ as in our basic method.

The important feature of the calculated $A(z)$ trajectories is their explosive behavior for all initial energies (as an example see the left panel of Fig. 4 ). At small $\Gamma<\Gamma_{c}$ a nucleus propagates with $A=$ const until large red-shifts $z \sim 1$ and then 'explodes' to $A_{0}$ on a scale of photo-disintegration lifetime $\tau_{a} \sim 10^{5}-10^{6} \mathrm{yr}$, see Eq. (12). It results in $z_{A} \approx z_{g}$ and $\Gamma_{A} \approx \Gamma_{g}$, where index $A$ marks the values at the threshold of an explosion. Therefore, the generation rate for $A$ nuclei approximately equals to that of primary nuclei $A_{0}: Q_{A}\left(\Gamma_{A}, z_{A}\right) \approx Q_{A_{0}}\left(\Gamma_{g}, z_{g}\right)$ with $z_{g} \approx z_{A}$ and $\Gamma_{g} \approx \Gamma_{A}$. However, the main attention in the paper is given to the exact calculation of $Q_{A}\left(\Gamma_{A}, z_{A}\right)$ from the number of particles conservation.

We describe first the combined method.

The space density of each species $a=A, p, A_{0}$ and their energy spectra are calculated using the kinetic equation

$$
\frac{\partial n_{a}\left(\Gamma_{a}, t\right)}{\partial t}-\frac{\partial}{\partial \Gamma_{a}}\left[b_{a}\left(\Gamma_{a}, t\right) n_{a}\left(\Gamma_{a}, t\right)\right]+\frac{n_{a}\left(\Gamma_{a}, t\right)}{\tau_{a}\left(\Gamma_{a}, t\right)}=Q_{a}\left(\Gamma_{a}, t\right)
$$

where $b_{a}=-d \Gamma_{a} / d t=\left(\beta_{\text {pair }}^{a}+\beta_{\text {ad }}^{a}\right) \Gamma_{a}$ is the rate of the Lorentz-factor loss, $\tau_{a}\left(\Gamma_{a}, z\right)$ is the photo-disintegration lifetime of particle $a$ (for $a=p, \tau_{a}=\infty$ ) and $Q_{a}\left(\Gamma_{a}, z\right)$ is the generation rate of particles $a$.

For secondary nuclei $A$ and protons, produced in the photo-disintegration processes, the generation rate $Q_{a}\left(\Gamma_{a}, z\right)$ is found from the conservation of the number of particles along a trajectory, $d N_{a}=d N_{g}$, where $d N_{a}$ is the number of produced particles $a$ and $d N_{g}$ is the number of generated primaries $A_{0}$ at acceleration. The relation between the generation rate of primaries $A_{0}$ and of secondary $A$ and $p$ is given by Eq. (60), where $z_{g}$ and $\Gamma_{g}$ are calculated using the evolution trajectories. The solutions of the kinetic equations (53) are found analytically.

An important physical quantity in our calculation is the critical Lorentz factor $\Gamma_{c}^{A}$ at epoch $z=0$. It is determined by the equality of the rates for changing of $\Gamma$ and $A: \quad \tau_{\text {dis }}^{-1}\left(\Gamma_{c}^{A}\right)=\tau_{\text {pair }}^{-1}\left(\Gamma_{c}^{A}\right)+H_{0}$, where $H_{0}$ is the Hubble constant, describing here the adiabatic energy loss, and indices 'dis' and 'pair' are related to photo-disintegration and pair-production lifetimes, respectively. This relation provides a stability condition for the $A$ nucleus at $z=0: \Gamma<\Gamma_{c}^{A}$. The critical Lorentz factor $\Gamma_{c}$ is a basic energy scale, which gives a key for understanding all processes considered here and which also explains some features in the calculated spectra.

Therefore, the combined method includes three elements of calculations: the kinetic equation, which gives the density of particles $n_{a}(\Gamma)$, the generation rate $Q_{a}\left(\Gamma_{a}, z_{a}\right)$ and the limits of integration, especially $z_{\min }^{a}$. The first two elements are reliable components of calculations, with $Q_{a}$ reliably evaluated with the help of the number of particles conservation; the third element $z_{\min }$ is less reliable since it involves additional consideration and assumptions.

The trajectory method of $n_{a}(\Gamma)$ calculation is less reliable because of the great uncertainties in the limits of integration $z_{\min }$ and $z_{\max }$ in Eq. (14).

The coupled kinetic equations (CKE) method is based on the set of kinetic equations (37), where the first one describes the primary nucleus $A_{0}$, the second $A_{0}-1$ etc down to the $A$ of interest. The solution of each preceding equation $n_{A^{\prime}}(\Gamma, z)$ gives the generation rate for a successive nucleus $A^{\prime}$ as $n_{A^{\prime}+1}(\Gamma, z) / \tau_{A^{\prime}+1}(\Gamma, z)$, and the long lifetime 
$\tau_{A^{\prime}+1}(\Gamma, z)$ at small $\Gamma$ and $z$ automatically provides $z_{\min }$ in the solution of a kinetic equation, while the lifetime $\tau_{A^{\prime}}$ provides, through the term $e^{-\eta}$, the upper limit. The $A(z)$ trajectories are formally not involved in this method, but actually they are. The term $n_{A_{0}} / \tau_{A_{0}}$ describes the disappearance of nuclei $A_{0}$ in the first equation of the Eqs. (37), but the same term appears in the second equation as generation of $\left(A_{0}-1\right)$ nuclei etc. Equation (51), which includes the product of terms with all $A^{\prime}$ from $A$ to $A_{0}$, demonstrates it more clearly. Indeed, this equation implies the evolution of $\Gamma(t)$ with $A^{\prime}=$ const regulated by $\exp \left(-t_{\text {prop }} / \tau_{A^{\prime}}\right)$. Then $A^{\prime}$ disappears, giving rise to the production term in the successive kinetic equation for $\left(A^{\prime}-1\right)$. However, there is an essential difference with the combined method. The product in Eq.(51) is time-ordered which, according to the theory of probabilities, means fluctuations taken into account.

As we indicated above the weakness of the combined method consists in the procedure of defining $z_{\mathrm{min}}$, while in the coupled kinetic equations this limit is a natural feature of the kinetic equation itself. The lower limit in the combined method is introduced by the stability condition given by Eq.(11), which is quite natural. However, in fact one can use the other definitions of stability. As a plausible example we consider an alternative possibility.

We used above in the combined method the cutoff in integration over $z$ imposing the condition $Q_{A}(\Gamma, t)=0$ at $t \geq t_{\max }$ in terms of the cosmological time $t$ (see the solution in terms of $t$ given by Eq. 45). This condition implies that at $t>t_{\max }(A+1)$-nucleus is stable. Let us introduce now this condition through the probability of $A+1$-decay:

$$
Q_{A}(\Gamma, t) \rightarrow\left[1-\exp \left(-\int_{t_{g}}^{t} \frac{d t^{\prime}}{\tau_{A+1}\left(\Gamma^{\prime}, t^{\prime}\right)}\right)\right] Q_{A}(\Gamma, t) .
$$

One can see that the factor introduced is the decay probability of the $A+1$ nucleus. It can be easily seen in the case $\tau_{A+1}=$ const, when this factor is $1-\exp \left(-t_{\text {prop }} / \tau_{A+1}\right)$, where $t_{\text {prop }}=t-t_{g}$ is the propagation time. The decay factor in Eq. (54) provides the soft cutoff of the generation function. However, this cutoff also differs from the one obtained in Eq. (52).

We compare now the numerical results in some details.

The two methods give numerically the same spectra for all nuclei at high energies and for primary nuclei at all energies. In particular both methods give high-energy fluxes of the secondary nuclei described by identical equations (49) and (23) with a recovery of the spectra at the highest energies due to an increasing $\tau_{A}(\Gamma)$.

The peaks in the spectra of the secondary nuclei located at $\Gamma_{c}$ in the combined method (see Fig. 10, right panel) coincide with the positions of peaks in the CKE method (see Fig. 15, left panel). This is a surprising result, because the critical Lorentz factor is not introduced in the CKE method. The fluxes above $\Gamma_{c}$ are the same, but they are much different below, which is mainly a result of the differences in $z_{\text {min }}$. The peculiar behaviour of ${ }^{4} \mathrm{He}$ at low energies in Fig. 10 (left panel), being confirmed by explosive trajectory calculation, is not seen in Fig. 15.

The fluxes of the secondary nuclei obey as a rule A-hierarchy: the heavier $A$, the larger the flux (see Figs. 10 and 15).

The main features of the secondary-proton spectra are the same in both methods. The $A$-associating proton spectra are the same for all $A$, and the total spectra are universal, i.e. do not depend on $A_{0}$. In the combined model this is a direct consequence of the explosive character of the trajectories. At low energies $\left(E<1 \times 10^{19} \mathrm{eV}\right)$ in CKE the $A$-associating proton spectra are different for various $A$ (see right panel in Fig. 15). This is related to a breaking of accuracy of the explosive trajectory approximation at low energies. 
The shape of the total proton spectrum is the same in both calculations. It has two spectral breaks. The high energy steepening is the usual GZK cutoff at energy $E \sim 5 \times 10^{19} \mathrm{eV}$. The low-energy steepening coincides with $\Gamma_{c}$. Below $\Gamma_{c}$ the adiabatic energy losses dominate and it explains the flat spectrum $n_{p}\left(E_{p}\right)$ there, i.e. the steep one in terms of $E^{3} n_{p}(E)$ spectrum as plotted in all figures here.

In the second paper of this series, the EBL background will be also included. In this case the high energy part of the nuclei spectra is determined by CMB, as considered in the present paper, while the low-energy part is affected mostly by Infrared, Visible and Ultra Violet radiations, which compose the EBL.

\section{Acknowledgements}

We thank Pasquale Blasi, Yurii Eroshenko and Askhat Gazizov for valuable discussions. This work is partially funded by the contract ASI-INAF I/088/06/0 for theoretical studies in High Energy Astrophysics and by the Gran Sasso Center for Astroparticle Physics (CFA) funded by European Union and Regione Abruzzo under the contract P.O. FSE Abruzzo 2007-2013, Ob. CRO. The work of SG is additionally funded by the grant of President of RF SS-3517.2010.2, VB and SG - by FASI grant under state contract 02.740.11.5092.

\section{A Generation rates of primary nuclei, secondary nuclei and secondary protons.}

We consider an expanding universe homogeneously filled by the sources of accelerated primary UHE nuclei $A_{0}$ with a generation rate per unit of comoving volume $Q_{A_{0}}(\Gamma, z)$ given by

$$
Q_{A_{0}}(\Gamma, z)=\frac{\left(\gamma_{g}-2\right)}{m_{N} A_{0}} \mathcal{L}_{0} \Gamma^{-\gamma_{g}}
$$

where $\gamma_{g}>2$ is the generation index, $m_{N}$ is the nucleon mass, and $\mathcal{L}_{0}$ is the source emissivity, i.e. the energy generated per unit of comoving volume and per unit time at $z=0$. In Eq. (55) $\Gamma_{\min } \sim 1$ is assumed. In all calculations in this paper we assume also the existence of a maximum energy of acceleration $E_{\max }^{\mathrm{acc}}=Z_{0} \times 10^{21} \mathrm{eV}$ (or $\Gamma_{\max }^{\mathrm{acc}}=$ $\left.\left(Z_{0} / A_{0}\right) \times 10^{12}\right)$ with the condition $Q_{A_{0}}\left(\Gamma_{g}\right)=0$ at $\Gamma_{g} \geq \Gamma_{\max }^{\text {acc }}$.

Propagating in the space, a nucleus $A_{0}$ experiences evolution, producing the secondary nuclei with different $A$ and we calculate now their generation rate $Q_{A}(\Gamma, z)$ per unit of comoving volume.

For calculation of the generation rates the evolution-trajectory formalism, developed in section 2.2, is used. The trajectories $A(z)=\mathcal{A}\left(\Gamma, A, z_{0}, z\right)$ and $\Gamma(z)=\mathcal{G}\left(\Gamma, A, z_{0}, z\right)$ in the backward time allows us to calculate the generation parameters $z_{g}$ and $\Gamma_{g}$, when $A(z)$ reaches $A_{0}$.

$A(z)$ can be calculated as continuous quantity, but we use the method of $A$-jump trajectories, as it is described in section 2.2. Namely, the trajectories are calculated assuming in Eqs. (9) for evolution of $\Gamma A=$ const, until $A$ reaches $A+1$ (or $A+2)$. Transition $A \rightarrow(A+1)$ is assumed to occur instantaneously. Accordingly, we use in this case the jump behaviour of $\beta_{\text {dis }}^{A}$. In fact both methods, continuous $A$ and jump $A$ approximations, give the same results. 
Since a recoil momentum in the processes of transition, e.g. $(A+1) \rightarrow A+N$, is negligibly small, one has approximate equality of Lorentz-factors of all three particles

$$
\Gamma_{A+1} \approx \Gamma_{A} \approx \Gamma_{N}
$$

The generation rate of secondary nuclei $A$ can be found from conservation of number of particles. Consider an allowed trajectory which connects $\left(\Gamma_{A}, z_{A}\right)$ state at generation $A$ with $\left(\Gamma_{g}, z_{g}\right)$ at generation of primary nucleus $A_{0}$. During time $d t_{g}$ at the epoch $z_{g}$ we have $d N_{A_{0}}$ primary nuclei generated with $\left(\Gamma_{g}, \Gamma_{g}+d \Gamma_{g}\right)$ :

$$
d N_{A_{0}}=Q_{A_{0}}\left(\Gamma_{g}, z_{g}\right) d \Gamma_{g} d t_{g} .
$$

All these nuclei appear as $d N_{A} \quad A$-nuclei with $\left(\Gamma_{A}, \Gamma_{A}+d \Gamma_{A}\right)$ during time $d t_{A}$ : $d N_{A}=d N_{A_{0}}$. Thus, we have

$$
Q_{A}\left(\Gamma_{A}, z\right) d \Gamma_{A} d t=Q_{A_{0}}\left(\Gamma_{g}, z_{g}\right) d \Gamma_{g} d t_{g}
$$

where the states $\left(\Gamma_{A}, z\right)$ and $\left(\Gamma_{g}, z_{g}\right)$ are connected by the allowed trajectory. From Eq. (58) and the time dilatation relation,

$$
d t_{g} / d t=(1+z) /\left(1+z_{g}\right)
$$

we obtain the generation rate of nuclei $A$ as:

$$
Q_{A}\left(\Gamma_{A}, z\right)=Q_{A_{0}}\left(\Gamma_{g}, z_{g}\right) \frac{1+z}{1+z_{g}} \frac{d \Gamma_{g}}{d \Gamma_{A}}
$$

with $d \Gamma_{g} / d \Gamma_{A}$ given in appendix $\mathrm{B}$,

As was emphasized above, the chain of equalities (58) is valid along the allowed trajectory, which breaks at some low $A$ and $z$, where $\tau_{A}$ becomes larger than $\tau_{\Gamma}$. If at $z=z_{c}$ $\gamma+(A+1) \rightarrow A+N$ is forbidden for all $\Gamma<\Gamma_{c}\left(z_{c}\right)$, then $Q_{A}\left(\Gamma_{A}, z_{A}\right)=0$ at $z_{A} \leq z_{c}$ and $\Gamma_{A} \leq \Gamma_{c}\left(z_{A}\right)$ (see also subsection 2.3 ). Starting from the critical redshift $z_{c}$ and below it $A$-nuclei are not produced and $(A+1)$-nuclei propagate remaining undestroyed.

Note, that for derivation we do not need stationary solution, and due to condition of homogeneity we do not need to discuss the spatial displacement of a particle.

Coming finally to the generation rate of nucleons produced in $(A+1) \rightarrow A+N$ transition, $Q_{p}^{A+1}\left(\Gamma_{p}, z\right)$, one may notice that this generation rate is the same that one for $A$ nuclei, since the Lorentz factor of both particles are equal and they are produce simultaneously.

$$
Q_{p}^{A+1}\left(\Gamma_{A}, z_{A}\right)=Q_{A_{0}}\left(\Gamma_{g}, z_{g}\right) \frac{1+z_{A}}{1+z_{g}} \frac{d \Gamma_{g}}{d \Gamma_{A}},
$$

where $z_{A}$ and $z_{g}$ is the redshifts of $A$ and $A_{0}$ production, and $\Gamma_{A}$ is the Lorentz factor of nucleus $A$ and nucleon. We do not distinguish nucleon and proton because UHE neutron decays fast, on the time scale of this problem, to proton.

\section{B Ratio of energy intervals at epochs of produc- tion and observation}

In this appendix we derive the ratio between Lorentz-factor intervals $d \Gamma_{g} / d \Gamma$ for a nucleus with variable atomic mass $A(z)$ propagating along the evolution trajectory $\Gamma_{g}(z)=$ 
$\mathcal{G}\left(A, \Gamma, z_{0}, z\right)$, where the first three indices describe the initial conditions and $z$ is the running redshift. The meaning of the generation index $g$ here is more general than the epoch of $A(z)$ evolution to $A_{0}$, though it includes this case, too. $\Gamma_{g}(z)$ means here the Lorentz factor that a nucleus has at the running epoch of evolution $z$ including $z_{g}$.

We use here the Lorentz-factor loss for a nucleus $A$ due to pair production as $b_{\text {pair }}^{A}(\Gamma, z)=$ $-d \Gamma / d t$, and express it through $b_{0}^{p}(\Gamma)=-d \Gamma / d t$ for proton at $z=0$. At epoch $z$ we have

$$
b_{\text {pair }}^{A}(\Gamma, z)=\frac{Z^{2}(z)}{A(z)}(1+z)^{2} b_{0}^{p}[(1+z) \Gamma],
$$

We introduce $\bar{k}=\left\langle Z^{2} / A^{2}\right\rangle$, assuming it be constant along the evolution trajectory $A(z)$, and thus using $Z^{2} / A=\bar{k} A(z)$.

The Lorentz factor of nucleus $A$ along the trajectory can be presented in a general form as

$$
\Gamma_{g}\left(t_{1}, t_{2}\right)=\Gamma+\int_{t_{1}}^{t_{2}} d t^{\prime}\left[\left(\frac{d \Gamma}{d t^{\prime}}\right)_{\text {ad }}+\left(\frac{d \Gamma}{d t^{\prime}}\right)_{\text {pair }}\right]
$$

Changing the variable $t$ to $z$ and using $d t=-d z /[(1+z) H(z)]$, we obtain

$$
\Gamma_{g}\left(z_{0}, z\right)=\Gamma+\int_{z_{0}}^{z} \frac{d z^{\prime}}{1+z^{\prime}} \Gamma_{g}\left(z^{\prime}\right)+\bar{k} \int_{z_{0}}^{z} d z^{\prime} \frac{1+z^{\prime}}{H\left(z^{\prime}\right)} A\left(z^{\prime}\right) b_{0}^{p}\left[\left(1+z^{\prime}\right) \Gamma_{g}\left(z^{\prime}\right)\right]
$$

Differentiating Eq. (64) in respect to $\Gamma$ and using

$$
\frac{d b_{0}^{p}\left(\Gamma^{\prime}\right)}{d \Gamma}=\frac{\partial b_{0}^{p}\left(\Gamma^{\prime}\right)}{\partial \Gamma^{\prime}} \frac{d \Gamma^{\prime}}{d \Gamma},
$$

one finds for the ratio of Lorentz-factor intervals $y(z) \equiv d \Gamma_{g}(z) / d \Gamma$ :

$$
y(z)=1+\int_{z_{0}}^{z} \frac{d z^{\prime}}{1+z^{\prime}} y\left(z^{\prime}\right)+\bar{k} \int_{z_{0}}^{z} d z^{\prime} \frac{\left(1+z^{\prime}\right)^{2} A\left(z^{\prime}\right)}{H\left(z^{\prime}\right)} y\left(z^{\prime}\right)\left(\frac{d b_{0}^{p}\left(\Gamma^{\prime}\right)}{d \Gamma^{\prime}}\right)_{\Gamma^{\prime}=\left(1+z^{\prime}\right) \Gamma_{g}\left(z^{\prime}\right)} .
$$

Differentiating Eq. (66) in respect to $z$ we obtain a differential equation for $y(z)$

$$
\frac{1}{y(z)} \frac{d y(z)}{d z}=\frac{1}{1+z}+\frac{\bar{k}}{H_{0}} \frac{(1+z)^{2} A(z)}{\sqrt{\Omega_{m}(1+z)^{3}+\Omega_{\Lambda}}}\left(\frac{d b_{0}^{p}\left(\Gamma^{\prime}\right)}{d \Gamma^{\prime}}\right)_{\Gamma^{\prime}=(1+z) \Gamma_{g}(z)} .
$$

The solution of Eq. (67) can be easily found as

$$
y(z) \equiv \frac{d \Gamma_{g}(z)}{d \Gamma\left(z_{0}\right)}=\frac{(1+z)}{\left(1+z_{0}\right)} \exp \left[\frac{\bar{k}}{H_{0}} \int_{z_{0}}^{z} d z^{\prime} \frac{\left(1+z^{\prime}\right)^{2} A\left(z^{\prime}\right)}{\sqrt{\Omega_{m}\left(1+z^{\prime}\right)^{3}+\Omega_{\Lambda}}}\left(\frac{d b_{0}^{p}\left(\Gamma^{\prime}\right)}{d \Gamma^{\prime}}\right)_{\Gamma^{\prime}=\left(1+z^{\prime}\right) \Gamma_{g}\left(z^{\prime}\right)}\right] .
$$

Equation (68) gives the ratio $d \Gamma_{g} / d \Gamma$, where $d \Gamma$ is the Lorentz-factor interval at the initial state $z_{0}$ (with most important case $z_{0}=0$ ), when a nucleus has fixed $A$ and Lorentz factor $\Gamma$, and $d \Gamma_{g}$ is the interval on the evolution trajectory at redshift $z$ when the atomic number is $A(z)$ and the Lorentz factor is $\Gamma_{g}(z)$.

In the applications we often need $d \Gamma_{g} / d \Gamma$ ratio for a fixed $A$. The most important case is the primary nuclei $A_{0}$. The ratio for fixed $A$ follows trivially from Eq. (68) substituting there $\bar{k} A\left(z^{\prime}\right)$ by constant value $Z^{2} / A$. For the proton case this value is 1 . 


\section{Solution to the kinetic equation}

In this appendix we derive the solution of the kinetic equation for secondary nuclei from which the solutions for primary nuclei and secondary protons can be easily obtained.

We consider the secondary nuclei $A$ being produced homogeneously in the space with the rate $Q_{A}(\Gamma, t)$ and then propagate as a nucleus species with the fixed (unchanged) $A$ until it is photo-disintegrated. The kinetic equation reads

$$
\frac{\partial n_{A}\left(\Gamma_{A}, t\right)}{\partial t}-\frac{\partial}{\partial \Gamma}\left[b_{A}(\Gamma, t) n_{A}(\Gamma, t)\right]+\frac{n_{A}(\Gamma, t)}{\tau_{A}(\Gamma, t)}=Q_{A}(\Gamma, t)
$$

where $b_{A}(\Gamma, t)$ is the rate of Lorentz factor loss given by

$$
b_{A}(\Gamma, t)=-\frac{d \Gamma}{d t}=\Gamma H(z)+\frac{Z^{2}}{A} b_{\text {pair }}^{p}(\Gamma, z),
$$

with $H(z)$ and $b_{\text {pair }}^{p}$ being the Hubble parameter at redshift $z$ and pair-production loss for proton, respectively. The time of photo-disintegration is given by $\tau_{A}^{-1}=A \beta_{\text {dis }}^{A}$ or by $\tau_{A}^{-1}=d A / d t$, see Eq. (7).

The characteristic equation for the kinetic equation (69) reads

$$
d \Gamma / d t=-b_{A}(\Gamma, t)
$$

With $\Gamma(t)$ taken on the characteristic in Eq. (69), the term $b_{A}(\Gamma, t) \partial n_{A}(\Gamma, t) / \partial \Gamma$ disappears and the kinetic equation (69) takes the form

$$
\frac{\partial n_{A}\left(\Gamma_{A}, t\right)}{\partial t}+n_{A}(\Gamma, t)\left[-\frac{\partial b_{\mathrm{pair}}^{A}(\Gamma, t)}{\partial \Gamma}-\frac{\partial b_{\mathrm{ad}}(\Gamma, t)}{\partial \Gamma}+\tau_{A}^{-1}(\Gamma, t)\right]=Q_{A}\left(\Gamma_{A}, t\right)
$$

Using the notation

$$
\begin{aligned}
& P_{1}(z) \equiv \partial b_{\mathrm{ad}}(z) / \partial \Gamma=H(z) \\
& P_{2}(z) \equiv \partial b_{\text {pair }}^{A}(\Gamma, z) / \partial \Gamma=\frac{Z^{2}}{A}(1+z)^{3}\left(\partial b_{0}^{p}\left(\Gamma^{\prime}\right) / \partial \Gamma^{\prime}\right)_{\Gamma^{\prime}=(1+z) \Gamma},
\end{aligned}
$$

and taking into account that with $\Gamma$ on the characteristic, time $t$ becomes the only variable, one obtains the solution of Eq. (72) as

$$
n_{A}(t)=\int_{t_{g}}^{t} d t^{\prime} Q_{A}\left(t^{\prime}\right) \exp \left[-\int_{t^{\prime}}^{t} d t^{\prime \prime}\left(-P_{1}\left(t^{\prime \prime}\right)-P_{2}\left(t^{\prime \prime}\right)+\tau_{A}^{-1}\left(t^{\prime \prime}\right)\right)\right] .
$$

Changing the integration variable $t$ to $z$ and using

$$
d t=-\frac{d z}{(1+z) H(z)}=-\frac{1}{H_{0}} \frac{d z}{(1+z) \sqrt{\Omega_{m}(1+z)^{3}+\Omega_{\Lambda}}}
$$

the solution can be written as

$$
\begin{gathered}
n_{A}(\Gamma, z=0)=\int_{0}^{z_{\max }} d z^{\prime} \frac{Q_{A}\left[\Gamma^{\prime}\left(\Gamma, z^{\prime}\right)\right]}{\left(1+z^{\prime}\right) H\left(z^{\prime}\right)} \times \\
\exp \left[\int_{0}^{z^{\prime}} d z^{\prime \prime} \frac{P_{1}\left(z^{\prime \prime}\right)}{\left(1+z^{\prime \prime}\right) H\left(z^{\prime \prime}\right)}\right] \exp \left[\int_{0}^{z^{\prime}} d z^{\prime \prime} \frac{P_{2}\left(z^{\prime \prime}\right)}{\left(1+z^{\prime \prime}\right) H\left(z^{\prime \prime}\right)}\right] \exp \left[-\int_{t^{\prime}}^{t_{0}} \frac{d t^{\prime \prime}}{\tau_{A}\left(\Gamma, t^{\prime \prime}\right)}\right]
\end{gathered}
$$


We keep the last integration over $t^{\prime \prime}$ to make clear the physical meaning of this integral as a suppression factor for the survival time of nucleus $A$. The upper limit $t_{0}$ is the age of the Universe.

Putting $P_{1}\left(z^{\prime \prime}\right)$ and $P_{2}\left(z^{\prime \prime}\right)$ from Eq. (73) into Eq. (76)), we find that the product of the first two exponents gives the ratio of energy intervals calculated in appendix $B$;

$$
\left(1+z^{\prime}\right) \exp \left[\frac{Z^{2}}{A} \frac{1}{H_{0}} \int_{0}^{z^{\prime}} d z^{\prime \prime} \frac{\left(1+z^{\prime \prime}\right)^{2}}{\sqrt{\Omega_{m}\left(1+z^{\prime \prime}\right)^{3}+\Omega_{\Lambda}}}\left(\frac{\partial b_{0}^{p}\left(\Gamma^{\prime \prime}\right)}{\partial \Gamma^{\prime \prime}}\right)_{\Gamma^{\prime \prime}=\left(1+z^{\prime \prime}\right) \Gamma_{g}\left(z^{\prime \prime}\right)}\right]=\frac{d \Gamma_{g}^{A}\left(z^{\prime}\right)}{d \Gamma^{A}} .
$$

Finally, we have

$$
n_{A}(\Gamma, z=0)=\int_{0}^{z_{\max }} d z^{\prime} \frac{Q_{A}\left[\Gamma^{\prime}\left(\Gamma, z^{\prime}\right)\right]}{\left(1+z^{\prime}\right) H\left(z^{\prime}\right)} \frac{d \Gamma^{\prime}}{d \Gamma} e^{-\eta\left(\Gamma^{\prime}, z^{\prime}\right)},
$$

where

$$
\eta\left(\Gamma^{\prime}, z^{\prime}\right)=\int_{t^{\prime}}^{t_{0}} \frac{d t^{\prime \prime}}{\tau_{A}\left(\Gamma^{\prime \prime}, t^{\prime \prime}\right)}=\int_{0}^{z^{\prime}} d z^{\prime \prime} \frac{1}{\left(1+z^{\prime \prime}\right) H\left(z^{\prime \prime}\right)} \frac{1}{\tau_{A}\left(\Gamma^{\prime \prime}, z^{\prime \prime}\right)} .
$$

In fact, in Eq. (78) one can put $z_{\max } \rightarrow \infty$ as upper limit, since it is regulated by the factor $\exp \left[-\eta\left(\Gamma^{\prime}, z^{\prime}\right)\right]$.

The equations (78) and (79) are valid for primary nuclei $\left(A_{0}, Z_{0}\right)$ and secondary protons; in the latter case one should put $\tau_{A} \rightarrow \infty$ and $\eta \rightarrow 0$.

\section{Comparison of the secondary nuclei and sec- ondary protons fluxes}

We perform here the analytic comparison of the fluxes of secondary protons and secondary nuclei at the same Lorentz factor $\Gamma$ in the most general form valid for kinetic equations and demonstrate that fluxes of the secondary protons are always higher.

The secondary nucleus $A$ and $A$-associating secondary nucleon $N$, born in the same decay $(A+1) \rightarrow A+N$, are twin brothers: they are born at the same redshift $z$ with the same Lorentz factor $\Gamma$ and with the same rate of production $Q_{A}(\Gamma, z)=Q_{p}^{A}(\Gamma, z)$ (we do not distinguish between neutron and proton because of the fast decay of the former). But one of the brothers, $A$-nucleus, lives short time $\tau_{A}$ and its flux is lower. We estimate here this effect analytically.

The flux of the $A$-associating secondary protons is discussed in sections 3.2 and 4 and in the most general form is given as

$$
n_{p}^{A}(\Gamma, 0)=\int_{0}^{z_{p}^{\max }} \frac{d z^{\prime}}{\left(1+z^{\prime}\right) H\left(z^{\prime}\right)} \frac{n_{A+1}\left(\Gamma^{\prime}, z^{\prime}\right)}{\tau_{A+1}\left(\Gamma^{\prime}, z^{\prime}\right)}\left(\frac{d \Gamma^{\prime}}{d \Gamma}\right)_{p},
$$

where $z_{0}=0$ is the initial condition, the generation term $Q_{p}^{A}(\Gamma, z)=Q_{A}(\Gamma, z)$ is written in the most general form, and $\Gamma^{\prime}\left(z^{\prime}\right)=G_{p}\left(\Gamma, 0, z^{\prime}\right)$ is the proton trajectory, on which $d \Gamma^{\prime} / d \Gamma$ ratio is given by

$$
\left(\frac{d \Gamma^{\prime}\left(z^{\prime}\right)}{d \Gamma}\right)_{p}=\left(1+z^{\prime}\right) \exp \left[\int_{0}^{z^{\prime}} \frac{d z^{\prime \prime}\left(1+z^{\prime \prime}\right)^{2}}{H\left(z^{\prime \prime}\right)}\left(\frac{d b_{0}^{p}(\tilde{\Gamma})}{d \tilde{\Gamma}}\right)_{\tilde{\Gamma}=\left(1+z^{\prime \prime}\right) \Gamma^{\prime \prime}}\right]
$$


Flux of the $A$-nuclei is calculated as

$$
n_{A}(\Gamma, 0)=\int_{0}^{z_{A}^{\max }} \frac{d z^{\prime}}{\left(1+z^{\prime}\right) H\left(z^{\prime}\right)} \frac{n_{A+1}\left(\Gamma^{\prime}, z^{\prime}\right)}{\tau_{A+1}\left(\Gamma^{\prime}, z^{\prime}\right)}\left(\frac{d \Gamma^{\prime}}{d \Gamma}\right)_{A} e^{-\eta\left(\Gamma^{\prime} \cdot z^{\prime}\right)},
$$

with

$$
\left(\frac{d \Gamma^{\prime}\left(z^{\prime}\right)}{d \Gamma}\right)_{A}=\left(1+z^{\prime}\right) \exp \left[\frac{A}{4} \int_{0}^{z^{\prime}} \frac{d z^{\prime \prime}\left(1+z^{\prime \prime}\right)^{2}}{H\left(z^{\prime \prime}\right)}\left(\frac{d b_{0}^{p}(\tilde{\Gamma})}{d \tilde{\Gamma}}\right)_{\tilde{\Gamma}=\left(1+z^{\prime \prime}\right) \Gamma^{\prime \prime}}\right]
$$

where we assumed $<Z / A\rangle=1 / 2$,

One may observe similarity of expressions for secondary protons and nuclei. It is easy to deduce inequality $n_{p}(\Gamma, 0)>n_{A}(\Gamma, 0)$ caused by two main reasons. The first one is given by $z_{p}^{\max }$ being considerably larger than $z_{A}^{\max }$, since the latter is limited by the decay lifetime $\tau_{A}$ (see factor $e^{-\eta}$ in Eq. 82). The second reason is connected with larger energy loss of nuclei to pair production $\beta_{A}(\Gamma)=(A / 4) \beta_{p}(\Gamma)$. It results in $\Gamma_{A}^{\prime}>\Gamma_{p}^{\prime}$ at the same $z^{\prime}$ in the integrand of Eq. (82), and thus to suppression of this integral. The larger $d \Gamma^{\prime} / d \Gamma$ for nuclei compensates partly the discussed flux inequality. Since this inequality is valid for every $A$, the total flux of the secondary protons also exceeds that of nuclei:

$$
\Sigma_{A} n_{p}^{A}(\Gamma, 0)>\Sigma_{A} n_{A}(\Gamma, 0) .
$$

At equal energies the nuclei flux should be shifted by factor $A$ to higher energies and in practical cases it exceeds the secondary proton flux.

\section{References}

[1] K. Greisen, Phys. Rev. Lett. 16748 (1966). G.T. Zatsepin and V.A. Kuzmin, Pisma Zh. Experim. Theor. Phys. 4114 (1966).

[2] V. Berezinsky and S. Grigorieva, A \& A 199, 1 (1988).

[3] V. Berezinsky, A. Gazizov, M. Kachelriess, Phys. Rev. Lett. 97, 231101 (2006).

[4] R. U. Abbasi [HiRes collaboration], Phys. Rev. Lett. 100,101101 (2008).

[5] T. Abu-Zayyad et al. [Telescope Array collaboration], arXiv:1205.5067.

[6] A. A. Watson, Highlight talk at ICRC 07, Nucl. Instr. and Meth. A588 (2008) 221.

[7] K-H Kampert, J.Phys.Conf.Ser. 120062002 (2008).

[8] Pierre Auger Collaboration, Phys. Rev. Lett. 101, 061101 (2008).

[9] Pierre Auger Collaboration, Phys. Lett. B685, 239 (2010).

[10] F. Salamida for the Pierre Auger Collaboration, Proc. 32th ICRC (Beijing, China) 2011, arXiv:1107.4809.

[11] V. Berezinsky, A. Gazizov and S. Grigorieva, Phys. Lett. B612 147 (2005).

[12] V. Berezinsky, A. Gazizov and S. Grigorieva, Phys. Rev. D74 043005 (2006).

[13] R. Aloisio, V. Berezisnky, P. Blasi, A. Gazizov, S. Grigorieva and B. Hnatyk, Astrop. Phys. 2776 (2007).

[14] V. Berezinsky, Journal of Physics: Conference Series 120012001 (2008).

[15] M. Nagano and A. Watson, Rev. Mod. Phys. 72, 689 (2000). 
[16] A. Watson, Nucl. Phys. B (Proc. Suppl.) 136, 290 (2004).

[17] T. Abu-Zayyad et al [HiRes collaboration] Phys. Rev. Lett. 92151101 (2004);

T. Abu-Zayyad et al [HiRes collaboration] Astrop. Phys. 23157 (2005).

[18] C. Jui et al. [Telescope Array Collaboration], Proc. APS DPF Meeting arXiv:1110.0133.

[19] T. Abu-Zayyad et al, Astrophys. J. 557, 686 (2001);

T. Abu-Zayyad et al, Phys. Rev. Lett., 84, 4276 (2000).

[20] A. V. Glushkov et al [Yakutsk collaboration], JETP Lett. 71, 97 (2000);

A. V. Glushkov and M. I. Pravdin, JETP Lett. 73, 115 (2001).

[21] D. Bird et al, Phys. Rev. Lett.,71, 4276 (1993);

D. J. Bird et al [Fly's Eye collaboration], Ap. J., 424, 491 (1994);

D. J. Bird et al, Ap.J. 441, 144 (1995).

[22] M. Ave et al, Astroparticle Phys. 1961 (2003).

[23] J. Abraham et al [Pierre Auger Collaboration] Phys. Rev. Lett., 104, 091101 (2010).

[24] R. Abbasi et al [HiRes Collaboration], Phys. Rev. Lett. 104161101 (2010).

[25] R. Aloisio, V. Berezinsky, P. Blasi, S. Ostapchenko, Phys. Rev. D77 (2008) 025007

[26] G. Giacinti, M. Kachelriess, D.V. Semikoz and G. Sigl, e-Print: arXiv:1104.1141 [astro-ph.HE];

G. Giacinti and D.V. Semikoz, Phys.Rev. D83 083002 (2011).

[27] M.P. Veron-Cetty and P. Veron, Astron. Astrophys. 455 (2006) 773.

[28] P. Abreu et al. [Pierre Auger Collaboration], Astropart.Phys. 34314 (2010);

J. Abraham et al. [Pierre Auger Collaboration], Science 318938 (2007).

[29] R. Aloisio and D. Boncioli, arXiv:1002.4134, accepted for publication in Astrop. Phys.

[30] R.U. Abbasi et al. [HiRes collaboration], Astropart.Phys.30 (2008) 175.

[31] D. Allard, A.V. Olinto and E. Parizot, A \& A 47359 (2007);

D. Allard, E. Parizot and A. V. Olinto, Astropart. Phys. 27 (2007) 61;

D. Allard, E. Parizot, E. Khan, S. Goriely and A.V. Olinto, A \& A 443 L29 (2005).

[32] R. Aloisio, V. Berezinsky and A. Gazizov, Astropart. Phys. 34620 (2011).

[33] A.Letessier-Selvon and T.Stanev, arXiv:1103.0031.

[34] K. Kotera, A. V. Olinto, arXiv:1101.4256,

[35] F. Aharonian, A. Bykov, E. Parizot, A. Watson, arXiv:1105.0131,

[36] F. W. Stecker, Phys. Rev. 180, 1264 (1969).

[37] J. L. Paget and F. W. Stecker, Proc. 14th ICRC 1975 Munich, 2734 (1975).

[38] V. S. Berezinsky and G. T. Zatsepin, Soviet Journal of Nuclear Physics, 13, 453 (1971).

[39] V.S. Berezinsky, S. I. Grigorieva, G.T. Zatsepin, Proc. 14th ICRC 1975 Munich, 2 711 (1975).

[40] A. M. Hillas, Proc, of 14th ICRC (Munich 1975) 2, 717 (1975).

[41] J.L. Puget, F.W. Stecker and J.H. Bredekamp, Astrophys. J. 205, 638 (1976).

[42] F.W. Stecker and M.H. Salamon, Astrophys. J. 512 (1999) 521. 
[43] E. Khan, S. Goriely, D. Allard, E. Parizot, T. Suomijarvi, A.J. Koning, S. Hilaire and M.C. Duijvestijn Astropart. Phys. 23191 (2005).

[44] K. Arisaka, G.B. Gelmini, M. Healy, O. Kalashev and J. Lee, JCAP 0712002 (2007);

[45] O.E. Kalashev, V.A. Kuzmin and D.V. Semikoz, astro-ph/9911035;

Mod. Phys. Lett. A 16, 2505 (2001);

O.E. Kalashev Ph.D. Thesis, INR RAS, 2003.

[46] M. Ahlers and A. M. Taylor, Phys. Rev. D82 123005 (2010);

D. Hooper, S. Sarkar, A. M. Taylor, Phys. Rev. D77 103007 (2008).

[47] M. Ahlers, L. Anchordoqui and S. Sarkar, Phys. Rev. D79 083009 (2009).

[48] D. Hooper and A. M. Taylor, Astrop. Phys. 33151 (2010);

D. Hooper, S. Sarkar, A. M. Taylor, Astrop. Phys. 27199 (2007);

D. Hooper, A. Taylor and S. Sarkar, Astropart. Phys. 23 (2005) 11.

[49] J. W. Elbert and P. Sommers, Ap. J. 441, 151 (1995).

[50] L.N. Epele and E. Roulet, JHEP 98109 (1998).

[51] G. Bertone, C. Isola, M. Lemoine and G. Sigl, Phys. Rev. D66 103003 (2002) 103003;

C. Isola, M. Lemoine, and G. Sigl, Phys. Rev. D 65023004 (2002);

G. Sigl, M. Lemoine, and P. Biermann, Astropart. Phys. 10141 (1999).

[52] T. Yamamoto, K. Mase, M. Takeda, N. Sakaki and M. Teshima, Astrop. Phys. 20 405 (2004).

[53] D. Allard, M. Ave, N. Busca, M. A. Malkan, A. V. Olinto, E. Parizot, F. W. Stecker and T. Yamamoto JCAP 9 (2006) 5;

M. Ave, N. Busca, A. V. Olinto, A. A. Watson and T. Yamamoto, Astropart. Phys. 23 (2005) 19;

M. Ave, N. Busca, A. V. Olinto, A. A. Watson and T. Yamamoto Nuclear Physics B 136159 (2004).

[54] E. Armengaud, G. Sigl and F. Miniati, Phys. Rev.D72 043009 (2005).

[55] G. Sigl and E. Armengaud, JCAP 5012 (2005).

[56] D. Harari, S. Mollerach and E. Roulet, JCAP 1112 (2006).

[57] L. A. Anchordoqui, H. Goldberg, D. Hooper, S. Sarkar and A. M. Taylor, Phys. Rev. D76 (2007) 123008.

[58] D. Allard, N.G. Busca, G. Decerprit, A.V. Olinto and E. Parizot, JCAP 0810 (2008) 033.

[59] R. Aloisio, D. Boncioli, A.F. Grillo, S. Petrera and F. Salamida, arXiv:1204.2970.

[60] V. Berezinsky, A. Gazizov and M. Kachelriess, Phys. Rev. Lett. 9723110 (2006).

[61] V. Berezinskii, S. Bulanov, V. Dogiel, V. Ginzburg, V.Ptuskin, Astrophysics of Cosmic Rays, North-Holland, 1990.

[62] D.N. Spergel et al. [WMAP Collaboration], Astrophys. J. Suppl. 148 (2003) 175.

D.N.Spergel et al., Ap.J.S. 170, 377 (2007).

[63] R. Aloisio and V. Berezinsky, Ap.J. 612, 900 (2004). 